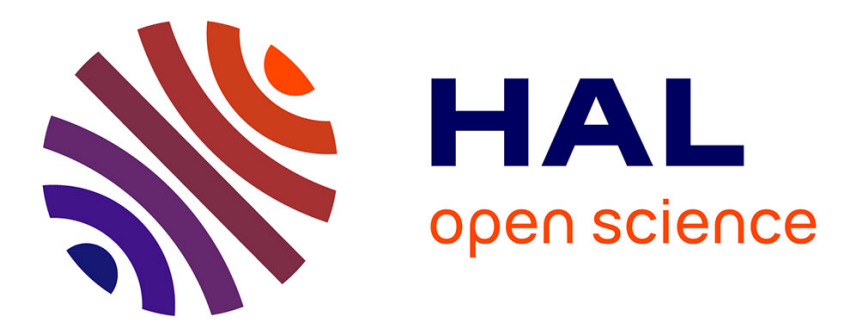

\title{
Diversification benefits under multivariate second order regular variation
}

\author{
Bikramjit Das, Marie Kratz
}

\section{To cite this version:}

Bikramjit Das, Marie Kratz. Diversification benefits under multivariate second order regular variation. 2017. hal-01520655

\section{HAL Id: hal-01520655 \\ https://essec.hal.science/hal-01520655}

Preprint submitted on 10 May 2017

HAL is a multi-disciplinary open access archive for the deposit and dissemination of scientific research documents, whether they are published or not. The documents may come from teaching and research institutions in France or abroad, or from public or private research centers.
L'archive ouverte pluridisciplinaire HAL, est destinée au dépôt et à la diffusion de documents scientifiques de niveau recherche, publiés ou non, émanant des établissements d'enseignement et de recherche français ou étrangers, des laboratoires publics ou privés. 


\title{
E \\ ESSEC \\ BUSINESS SCHOOL
}

\section{DIVERSIFICATION BENEFITS UNDER MULTIVARIATE SECOND ORDER REGULAR VARIATION}

\author{
RESEARCH CENTER \\ BIKRAMJIT DAS AND MARIE KRATZ \\ ESSEC WORKING PAPER 1706
}

APRIL 2017 


\title{
Diversification benefits under multivariate second order regular variation
}

\author{
BIKRAMJIT DAS and MARIE KRATZ
}

Singapore University of Technology and Design

8 Somapah Road, Singapore 487372

E-mail: bikram@sutd.edu.sg

ESSEC Business School, CREAR

avenue Bernard Hirsch BP 50105

Cergy-Pontoise 95021 Cedex, France

E-mail: kratz@essec.edu

\begin{abstract}
We analyze risk diversification in a portfolio of heavy-tailed risk factors under the assumption of second order multivariate regular variation. Asymptotic limits for a measure of diversification benefit are obtained when considering, for instance, the value-at-risk. The asymptotic limits are computed in a few examples exhibiting a variety of different assumptions made on marginal or joint distributions. This study ties up existing related results available in the literature under a broader umbrella.
\end{abstract}

AMS 2000 subject classifications: 60G70, 60E05, 62E20, 62P05, 91B30

Keywords: asymptotic theory, diversification benefit, heavy tail, risk concentration, second order regular variation, value-at-risk.. 


\section{Introduction}

An important issue in risk management is assessing the effects of adding an investment to a portfolio of risk factors (time series of returns) and understanding how this aggregate risk relates to the individual risk factors. Broadly studied under the labels of risk concentration or risk diversification, the past couple of decades have seen tremendous developments in the understanding of this topic. Our interest is in a portfolio of risk factors that are heavy-tailed, where adequate care is necessary to study the aggregation of the risk factors; see Dacorogna et al. (2015), Embrechts et al. (2002), Ibragimov et al. (2011), Puccetti and Rüschendorf (2013) for detailed discussions on diversification, especially under heavy-tailed returns.

In this paper, we consider the particular risk measure value-at-risk. Recall that for a random variable (risk factor) $X$ with distribution function $F$, the value-at-risk at level $0<\beta<1$ is defined as

$$
\operatorname{VaR}_{\beta}(X):=\inf \{y \in \mathbb{R}: \mathbb{P}(X \leq y) \geq \beta\}=F^{\leftarrow}(\beta) .
$$

Consider a portfolio of risk factors $\boldsymbol{X}=\left(X_{1}, \ldots, X_{d}\right)$. We assume for this paper that $X_{1}, \ldots, X_{d}$ are identically distributed (homogeneous) non-negative random variables with a common continuous distribution function. The behavior of the sum

$$
S_{d}=X_{1}+\ldots+X_{d}
$$

and its value-at risk $\operatorname{VaR}_{\beta}\left(S_{d}\right)$ have been studied under various assumptions, either on the marginal distribution $F$ (where $X_{1} \sim F$ ) or on the dependence structure of $\boldsymbol{X}$. If $X_{1}, \ldots, X_{d}$ are independent and identically distributed (iid) with a regularly varying tail distribution with tail parameter $\alpha$, that is, $\bar{F}=1-F \in \mathcal{R} \mathcal{V}_{-\alpha}$ (see Section 1.1 for details) then it is well-known that $\operatorname{VaR}\left(S_{d}\right)$ is asymptotically sub-additive or super-additive according as $\alpha>1$ or $\alpha<1$ (see Degen et al. (2010), Embrechts et al. (2009)) and an accurate estimation for high threshold has been proposed in Kratz (2014). Since an assumption of regular variation provides only a first order approximation, researchers have studied second order behaviors of $\operatorname{VaR}_{\beta}\left(S_{d}\right)$ under a second order regular variation assumption on $\bar{F}$; see Degen et al. (2010), Mao and Hu (2013). Furthermore, there has been a series of studies on the asymptotic behavior of the tail of $S_{d}$ and $\operatorname{VaR}_{\beta}\left(S_{d}\right)$ under specific copula assumptions on the dependence structure of $\boldsymbol{X}$; see Albrecher et al. (2010), Alink et al. (2004), Barbe et al. (2006), Kortschak (2012), Sun and Li (2010); or by providing risk bounds under assumptions on marginal densities, see Peng et al. (2013), Puccetti and Rüschendorf (2013).

In this paper we work under the assumption that $\boldsymbol{X}=\left(X_{1}, \ldots, X_{d}\right)$ is multivariate second order regularly varying. This assumption encompasses examples of independent, asymptotically independent, as well as dependent risk factors and brings together a variety of marginal and dependence assumptions on the joint distribution of $F$ under one broad umbrella. The structure of the paper is as follows. In Section 1.1 we briefly collate notations to be used in the paper. The various notions of regular variation both first order and second order as well as univariate and multivariate are described and discussed in Section 1.2. In Section 2 we discuss risk aggregation under multivariate second order regular variation. The main results of risk diversification for value-at-risk are discussed in Section 3. Some examples to illustrate our results are given in Section 4. We provide conclusions and future directions in Section 5. The appendix in Section 6 recalls, for completeness, results from Resnick (2002) that characterize second order regular variation in terms of vague convergence of signed measures, and which are used in our results. 


\subsection{Notations}

A brief summary of some notation and concepts used in this paper are provided here. We use bold letters to denote vectors, with capital letters for random vectors and small letters for non-random vectors, e.g., $\boldsymbol{y}=\left(y_{1}, y_{2}\right) \in \mathbb{R}^{2}$. We also define $\mathbf{0}=(0,0)$ and $\boldsymbol{\infty}=(\infty, \infty)$. Vector operations are always understood component-wise, e.g., for vectors $\boldsymbol{x}$ and $\boldsymbol{y}, \boldsymbol{x} \leq \boldsymbol{y}$ means $x_{i} \leq y_{i}$ for all $i$. For a constant $k \in \mathbb{R}$ and a set $A \subset \mathbb{R}^{d}$, we denote by $k A:=\{k \boldsymbol{x}: \boldsymbol{x} \in A\}$. Some additional notation follows with explanations that are amplified in subsequent sections. Detailed discussions are in the references.

$\begin{array}{ll}\mathbb{E}^{*} & \text { A compactified version of a nice subset of the finite-dimensional Euclidean } \\ & \text { space, often denoted } \mathbb{E} \text { with different subscripts and superscripts, as required. } \\ & \text { For example, we often denote } \mathbb{E}=[0, \infty]^{d} \backslash\{\mathbf{0}\} \text { and } \mathbb{E}_{0}=(0, \infty]^{d} \text {. } \\ \mathcal{B}\left(\mathbb{E}^{*}\right) & \text { The Borel } \sigma \text {-field of the subspace } \mathbb{E}^{*} \text {. } \\ \aleph & \text { The set }\{\boldsymbol{x} \in \mathbb{E}:\|\boldsymbol{x}\|=1\} \text {, where }\|\cdot\| \text { denotes the Euclidean norm in } \mathbb{R}^{d} \text {. } \\ \mathbb{M}_{+}\left(\mathbb{E}^{*}\right) & \text { The class of Radon measures on Borel subsets of } \mathbb{E}^{*} \text {. } \\ \stackrel{v}{\rightarrow} & \text { vague convergence of measures, often on } \mathbb{M}_{+}\left(\mathbb{E}^{*}\right) ; \text { see Resnick }(2007) . \\ f^{\leftarrow} & \text { The left-continuous inverse of a monotone function } f \text {. } \\ & \text { For a non-decreasing function } f, \text { we have } f^{\leftarrow}(x)=\inf \{y: f(y) \geq x\} . \\ & \text { For a non-increasing function } g, \text { we have } g^{\leftarrow}(x)=\inf \{y: g(y) \leq x\} . \\ & \text { The class of regularly varying functions with index } \rho \in \mathbb{R}, \text { that is, functions } \\ & f: \mathbb{R}_{+} \rightarrow \mathbb{R}_{+} \text {satisfying limt } \mathcal{V}_{\rho} \quad f(t x) / f(t)=x^{\rho}, \text { for } x>0 ; \text { see Bingham et al. } \\ & (1989), \text { de Haan }(1970), \text { de Haan and Ferreira }(2006), \operatorname{Resnick}(2008) .\end{array}$

\subsection{Preliminaries}

Regular variation often forms the basis for studying heavy-tailed distributions. In this section we recall definitions and properties of regular variation and second order regular variation in both univariate and multivariate case (Bingham et al., 1989, de Haan, 1970, de Haan and Ferreira, 2006, Resnick, 2002, 2008). Definition 1.5 for multivariate second order regular variation forms the key assumption of our models for this paper. We also define the related concept of hidden regular variation in Definition 1.6, which may be used to generate models possessing multivariate second order regular variation as seen in Example 4.3.

Recall that a function $f: \mathbb{R}_{+} \rightarrow \mathbb{R}_{+}$is regularly varying (at $\infty$ ) with parameter $\rho \in \mathbb{R}$ if

$$
\lim _{t \rightarrow \infty} f(t x) / f(t)=x^{\rho}
$$

for any $x>0$. We write $f \in \mathcal{R} \mathcal{V}_{\rho}$.

\subsubsection{Regular variation in one-dimension}

A large class of heavy-tailed distributions belonging to the maximum domain of attraction of the Fréchet distribution corresponds to the paradigm of regular variation of the tail of the distribution. 
Definition 1.1 (Regular variation, Bingham et al. (1989)). A random variable $X$ with distribution function $F$ has regularly varying (right) tail with index $\alpha \geq 0$ if $\bar{F}=1-F \in \mathcal{R} \mathcal{V}_{-\alpha}$. Alternatively, we say that there exists a function $b: \mathbb{R}_{+} \rightarrow \mathbb{R}_{+}$with $b(t) \uparrow \infty$ as $t \rightarrow \infty$ such that

$$
\lim _{t \rightarrow \infty} t \mathbb{P}[X>b(t) x]=x^{-\alpha} .
$$

In terms of vague convergence we can think of convergence in the space $(0, \infty]$, where

$$
\frac{\mathbb{P}\left[t^{-1} X \in \cdot\right]}{\mathbb{P}[X>t]} \underset{t \rightarrow \infty}{\stackrel{v}{\longrightarrow}} \mu_{\alpha}(\cdot)
$$

with $\mu_{\alpha}(d x)=\alpha x^{-\alpha-1} d x$. We write $\bar{F} \in \mathcal{R} \mathcal{V}_{-\alpha}$ or, by abuse of notation, $X \in \mathcal{R} \mathcal{V}_{-\alpha}$.

A consequence of the definition is that $b \in \mathcal{R} \mathcal{V}_{1 / \alpha}$ and a natural choice is $b(t)=(1 / F)^{\leftarrow}(t)$. For example, Pareto, Fréchet, Stable or Burr distribution with parameter $\alpha$ have $\mathcal{R} \mathcal{V}_{-\alpha}$ tail distributions (see e.g. Embrechts et al. (1997)).

Furthermore, often some distributions with regularly varying tails have a second order property that is not captured by the scaling in the definition of regular variation. The Pareto-Lomax distribution is one such example, analyzed below. The following definition provides one approach to studying such distributions.

Definition 1.2 (Second order regular variation; de Haan and Resnick (1993), Resnick (2002),§3). $A$ random variable $X$ with distribution function $F$ such that $\bar{F} \in \mathcal{R V}_{-\alpha}$ with $\alpha \geq 0$, possesses second order regular variation with parameter $\rho \leq 0$ if there exist functions $b(\cdot) \in \mathcal{R}_{1 / \alpha}$ and $A(t) \underset{t \rightarrow \infty}{\rightarrow} 0$ that is ultimately of constant sign, $|A(\cdot)| \in \mathcal{R} \mathcal{V}_{\rho}$ with $\rho \leq 0$ and $c \neq 0$ such that

$$
\frac{t \bar{F}(b(t) x)-x^{-\alpha}}{A(b(t))} \underset{t \rightarrow \infty}{\longrightarrow} c x^{-\alpha} \frac{x^{\rho}-1}{\rho}=: H(x) .
$$

The right hand side of (1.2) is interpreted as $H(x)=c \log (x)$ when $\rho=0$. We write $\bar{F} \in$ $2 \mathcal{R} \mathcal{V}_{-\alpha, \rho}(b, A, H)$ or, by abuse of notation, $X \in 2 \mathcal{R} \mathcal{V}_{-\alpha, \rho}(b, A, H)$. The arguments in the brackets are often dropped for simplicity.

Remark 1.3. An equivalent representation of second order regular variation is the following: $\bar{F} \in 2 \mathcal{R} \mathcal{V}_{-\alpha, \rho}(A, H)$ if there exists an ultimately positive or negative function $A$ with $A(t) \underset{t \rightarrow \infty}{\rightarrow} 0$ such that

$$
\lim _{t \rightarrow \infty} \frac{\frac{\bar{F}(t x)}{\bar{F}(t)}-x^{-\alpha}}{A(t)}=c x^{-\alpha} \frac{x^{\rho}-1}{\rho}=: H(x)
$$

for some constant $c \neq 0$ and parameters $\alpha>0, \rho \in \mathbb{R}$. The parameters $\alpha, \rho$ of course remain the same in both definitions. With a choice of $b(t)=(1 / \bar{F})^{\leftarrow}(t)$, the functions $A$ and $H$ also coincide.

Example 1.1. Consider the Pareto-Lomax distribution function for $\alpha>0$ given by $\bar{F}(x)=$ $(1+x)^{-\alpha}, x>0$. Choosing $b(t)=(1 / F)^{\leftarrow}(t)=t^{1 / \alpha}-1$ and $A(t)=(1+t)^{-1}$, we obtain

$$
\lim _{t \rightarrow \infty} \frac{t \bar{F}(b(t) x)-x^{-\alpha}}{A(b(t))}=\lim _{t \rightarrow \infty} \frac{t\left(1+\left(t^{1 / \alpha}-1\right) x\right)^{-\alpha}-x^{-\alpha}}{(1+t)^{-1}}=-\alpha x^{-\alpha}\left(x^{-1}-1\right)=: H(x) .
$$

Hence $\bar{F} \in 2 \mathcal{R} \mathcal{V}_{-\alpha,-1}(b, A, H)$. 


\subsubsection{Regular variation in multiple dimensions}

Multivariate regular variation facilitates the study of jointly heavy-tailed random variables and is a natural extension to Definition 1.1. The following definitions explain multivariate regular variation as well as second order regular variation for joint tail distributions of random variables. The notion of vague convergence of measures is used for convergence of measures on the non-negative Euclidean orthant $\mathbb{R}_{+}^{d}$ and its subsets; see Resnick (2007) for further details.

Definition 1.4 (Multivariate regular variation, Resnick (2007)). Suppose $\boldsymbol{X}=\left(X_{1}, \ldots, X_{d}\right)$ is a random vector in a cone $[0, \infty)^{d}$. Then $\boldsymbol{X}$ is multivariate regularly varying with limit measure $\nu$, if there exist $b(t) \uparrow \infty$ and a Radon measure $\nu \neq 0$ such that, on $\mathbb{E}=[0, \infty]^{d} \backslash\{\mathbf{0}\}$,

$$
t \mathbb{P}\left(\frac{\boldsymbol{X}}{b(t)} \in \cdot\right) \underset{t \rightarrow \infty}{\rightarrow} \nu(\cdot) \quad \text { on } \quad \mathbb{M}_{+}(\mathbb{E})
$$

We write $\boldsymbol{X} \in \mathcal{M R \mathcal { V }}{ }_{-\alpha}(b)$

It is easy to check that $\nu(\cdot)$ is homogeneous in the sense that, for $\alpha \geq 0$ and relatively compact $A \subset \mathbb{E}$,

$$
\nu(c A)=c^{-\alpha} \nu(A), \quad c>0 .
$$

We can also check that $b(\cdot) \in \mathcal{R} \mathcal{V}_{1 / \alpha}$.

Definition 1.5 (Second order multivariate regular variation, Resnick (2002)). Suppose $\boldsymbol{X} \in$ $\mathcal{M R} \mathcal{V}_{-\alpha}(b)$ and there exists $A(t) \underset{t \rightarrow \infty}{\rightarrow} 0$ that is ultimately of constant sign with $|A(\cdot)| \in \mathcal{R} \mathcal{V}_{\rho}, \rho \leq 0$ such that

$$
\frac{t \mathbb{P}\left(\frac{\boldsymbol{X}}{b(t)} \in[\mathbf{0}, \boldsymbol{x}]^{c}\right)-\nu\left([\mathbf{0}, \boldsymbol{x}]^{c}\right)}{A(b(t))} \underset{t \rightarrow \infty}{\rightarrow} H(\boldsymbol{x})
$$

locally uniformly in $\boldsymbol{x} \in(0, \infty]^{d} \backslash\{\infty\}$, where $H(\boldsymbol{x})$ is a function that is non-zero and finite. Then $\boldsymbol{X}$ is second order regularly varying with parameters $\alpha \geq 0$ and $\rho \leq 0$. We write $\boldsymbol{X} \in$ $2 \mathcal{M R} \mathcal{V}_{-\alpha, \rho}(b, A, \nu, H)$; some or all of the parameters may be omitted according to the context.

Observe that putting $d=1$ in Definitions 1.4 and 1.5 gives us back the univariate versions Definitions 1.1 and 1.2 . In order to use (1.5) in terms of vague convergence of signed measures, we impose further conditions on the distribution $F$ of $\boldsymbol{X}$ as aptly noted in (Resnick, 2002, Section 4). Appropriate conditions, used in this paper to obtain the results, are described in Assumptions 1 and 2 in the Appendix (Section 6).

The connection between second order regular variation and hidden regular variation has been discussed in detail in Resnick (2002). Recall that a $d$-dimensional non-negative random vector $\boldsymbol{X} \in \mathcal{M R \mathcal { V }} \mathcal{- \alpha}(b, \nu)$ possesses asymptotic independence if $\nu\left((0, \infty]^{d}\right)=0$, meaning that, the limit measure $\nu$ concentrates only on the coordinate axes. In the presence of such a phenomenon of asymptotic independence, hidden regular variation, as described below, is sometimes observed. 
Definition 1.6. A random vector $\boldsymbol{X} \in \mathbb{R}_{+}^{d}$ has hidden regular variation if $\boldsymbol{X} \in \mathcal{M R \mathcal { V }}{ }_{-\alpha}(b)$ and there exist a function $b_{0}$ and a limit measure $\nu_{0} \neq 0$ on $\mathbb{E}_{0}=(0, \infty]^{d}$, such that $\lim _{t \rightarrow \infty} b(t) / b_{0}(t)=\infty$ and for $A \in \mathcal{B}\left(\mathbb{E}_{0}\right)$,

$$
\lim _{t \rightarrow \infty} t \mathbb{P}\left(\frac{\boldsymbol{X}}{b_{0}(t)} \in A\right)=\nu_{0}(A)
$$

Many models with hidden regular variation also happen to exhibit second order regular variation; specifically we can look at additive models and mixture models; for further details see Das and Resnick (2015), Weller and Cooley (2014). Example 4.3 in Section 4 is created in this way.

\section{Aggregation under multivariate second order regular variation}

In order to aggregate multiple risks factors (with the same marginal distribution or at least equivalent tail order), multivariate regular variation helps in providing justification for sub- or superadditivity; see Degen and Embrechts (2011), Embrechts et al. (2009). We observe here that further structure and intuition can be provided by assuming second order regular variation. The key idea in this section is to relate second order regular variation of the multivariate kind with the same of the univariate kind. This eventually helps us in evaluating risk measures for sums of homogeneous random factors with different dependence structures.

Aggregation of risk under multivariate regular variation is relatively straightforward to check. For example, assuming that $\boldsymbol{X} \in \mathcal{M R \mathcal { V }}{ }_{-\alpha}(b)$ with identical marginals $X_{i} \sim F$, then, using the definition, we can check that, if $S_{d}:=\sum_{i=1}^{d} X_{i} \sim F_{S_{d}}$ for $d \geq 2$, then $F_{S_{d}} \in \mathcal{R} \mathcal{V}_{-\alpha}$ with the same function $b(\cdot)$ as in the Definition 1.4. Moreover, $b(\cdot)$ need not be asymptotically equivalent to $\left(1 / F_{S_{d}}\right)^{\leftarrow}(\cdot)$. The following proposition extends this implication to the case where $\boldsymbol{X}$ possesses second order regular variation.

Proposition 2.1. Assume $\boldsymbol{X} \in 2 \mathcal{M R} \mathcal{V}_{-\alpha, \rho}(b, A, \nu, H)$ with functions $b(t) \uparrow \infty$ and $A(t) \rightarrow 0$, as $t \rightarrow \infty$, so that Condition (1.5) holds in terms of vague convergence of signed measures, under either Assumption 1 or Assumption 2 (see Appendix). Then

$$
\begin{gathered}
S_{d} \in 2 \mathcal{R}_{-\alpha, \rho}\left(b_{d}, A_{d}, H_{d}\right), \text { where } \\
\begin{cases}b_{d}(t) & :=\left(\nu\left(\Gamma_{d}\right)\right)^{1 / \alpha} b(t), \\
A_{d}(t) & :=A\left(\left(\nu\left(\Gamma_{d}\right)\right)^{-1 / \alpha} t\right), \\
H_{d}(x) & :=\chi\left(x\left(\nu\left(\Gamma_{d}\right)\right)^{1 / \alpha} \Gamma_{d}\right)=c_{d} x^{-\alpha} \frac{x^{\rho}-1}{\rho}, \quad \text { with } \quad c_{d}=\frac{\rho 2^{\alpha}}{2^{\rho}-1} \chi\left(2\left(\nu\left(\Gamma_{d}\right)\right)^{1 / \alpha} \Gamma_{d}\right),\end{cases}
\end{gathered}
$$

where $\chi$ is defined as $\chi\left([\mathbf{0}, \boldsymbol{x}]^{c}\right)=H(\boldsymbol{x})$, and

$$
\Gamma_{d}:=\left\{z \in \mathbb{R}_{+}^{d}: z_{1}+z_{2}+\ldots+z_{d}>1\right\}
$$

By construction we have $A_{d}\left(b_{d}(t)=A(b(t))\right.$. We also extend the notation for $\Gamma_{d}$ in $(2.2)$ defined for $d \geq 2$ to the case where $d=1$ as

$$
\Gamma_{1}:=\left\{z \in \mathbb{R}_{+}^{d}: z_{1}>1\right\}
$$


Remark 2.2.

(i) Assuming $\boldsymbol{X} \in 2 \mathcal{M R \mathcal { V }}{ }_{-\alpha, \rho}(b, A, \nu, H)$ along with either Assumption 1 or Assumption 2, implies that $\chi\left(k \Gamma_{d}\right) \neq 0$ for some $k>0$ and hence the constant $c_{d}$ is non-zero. Nevertheless, we can construct examples where $c_{d}=0$, yet $\boldsymbol{X} \in 2 \mathcal{M R \mathcal { V }}{ }_{-\alpha, \rho}(b, A, \nu, H)$ holds; see Example 2.2. Both Assumptions 1 and 2 require that the marginal distributions are identical, which is violated in Example 2.2.

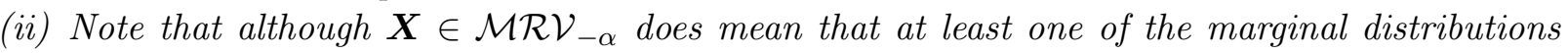
is $\mathcal{R} \mathcal{V}_{-\alpha}$, such an implication is not necessarily true for a $2 \mathcal{M R \mathcal { V }}$ condition. Assuming $\boldsymbol{X} \in$ $2 \mathcal{M R} \mathcal{V}_{-\alpha, \rho}$ does not necessarily imply that one of the components is $2 \mathcal{R} \mathcal{V}$. For instance, if the components of $\boldsymbol{X}$ are all iid $F$ that is Pareto( $\alpha$-type 1, meaning $F(x)=1-x^{-\alpha}, x>1$ and $\alpha>0$, then $\boldsymbol{X} \in 2 \mathcal{M R \mathcal { V }}{ }_{-\alpha}$, although none of the margins are $2 \mathcal{R} \mathcal{V}$.

(iii) The reverse implication of Proposition 2.1, properly worded, would say that, if any convex combination of $\boldsymbol{X}$ is $2 \mathcal{R} \mathcal{V}$ then $\boldsymbol{X} \in 2 \mathcal{M R} \mathcal{V}$. We conjecture that such a result would require further conditions on the random variables to hold. See Basrak et al. (2002) for the conditions that allows this to happen for regularly varying random vectors (not necessarily $2 \mathcal{R} \mathcal{V}$

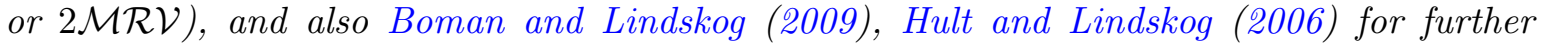
investigation.

Proof of Proposition 2.1. Since $\boldsymbol{X} \in 2 \mathcal{M R \mathcal { V }}{ }_{-\alpha, \rho}(b, A, \nu, H)$ and either Assumption 1 or Assumption 2 holds (this also ensures identical marginals), we have

$$
\mu_{t}^{ \pm} \stackrel{v}{\rightarrow} \chi^{ \pm}, \quad \text { on } \quad \mathbb{E}
$$

where, for $t>0, \mu_{t}^{+}, \mu_{t}^{-}, \chi^{+}, \chi^{-}$are positive Radon measures with $\mu_{t}=\mu_{t}^{+}-\mu_{t}^{-}$and $\chi=\chi^{+}-\chi^{-}$, $\chi: A \rightarrow \mathbb{R}$ for a Borel subset $A \subset[0, \infty)^{d} \backslash\{\mathbf{0}\}$ defined by $\chi\left([\mathbf{0}, \boldsymbol{x}]^{c}\right)=H(\boldsymbol{x})$ and $\mu_{t}$ defined in (6.7). Hence we have

$$
\mu_{t}\left(\Lambda_{d}\right)=\frac{t \mathbb{P}\left(\frac{\boldsymbol{X}}{b(t)} \in \Lambda_{d}\right)-\nu\left(\Lambda_{d}\right)}{A(b(t))} \underset{t \rightarrow \infty}{\rightarrow} \chi\left(\Lambda_{d}\right)
$$

for any relatively compact $\Lambda_{d} \subset \mathbb{E}$. Define $b_{d}(t)=\left(\nu\left(\Gamma_{d}\right)\right)^{1 / \alpha} b(t)$. Then, for $x>0$,

$$
t \mathbb{P}\left(\frac{S_{d}}{b_{d}(t)}>x\right)=t \mathbb{P}\left(\frac{\boldsymbol{X}}{b(t)} \in x\left(\nu\left(\Gamma_{d}\right)\right)^{1 / \alpha} \Gamma_{d}\right) \underset{t \rightarrow \infty}{\longrightarrow} \nu\left(x\left(\nu\left(\Gamma_{d}\right)\right)^{1 / \alpha} \Gamma_{d}\right)=x^{-\alpha}
$$

using (1.4). Now, let $A_{d}(t)=A\left(\left(\nu\left(\Gamma_{d}\right)\right)^{-1 / \alpha} t\right)$ for $t>0$. Then by applying (2.3), we get for $x>0$,

$$
\begin{aligned}
\frac{t \mathbb{P}\left(\frac{S_{d}}{b_{d}(t)}>x\right)-x^{-\alpha}}{A_{d}\left(b_{d}(t)\right)} & =\frac{t \mathbb{P}\left(\frac{\boldsymbol{X}}{b(t)} \in x\left(\nu\left(\Gamma_{d}\right)\right)^{1 / \alpha} \Gamma_{d}\right)-\nu\left(x\left(\nu\left(\Gamma_{d}\right)\right)^{1 / \alpha} \Gamma_{d}\right)}{A(b(t))} \\
& =\mu_{t}\left(x\left(\nu\left(\Gamma_{d}\right)\right)^{1 / \alpha} \Gamma_{d}\right) \underset{t \rightarrow \infty}{\longrightarrow} \chi\left(x\left(\nu\left(\Gamma_{d}\right)\right)^{1 / \alpha} \Gamma_{d}\right) .
\end{aligned}
$$

Defining $H_{d}(x):=\chi\left(x\left(\nu\left(\Gamma_{d}\right)\right)^{1 / \alpha} \Gamma_{d}\right)$, we know that $H_{d}$ is not identically zero by the assumption $\chi\left(k \Gamma_{d}\right) \neq 0$ for some $k>0$. Hence using Remark 1.3 and Theorem 2.3.9 in de Haan and Ferreira (2006), we can represent $H_{d}$ as follows: for $x>0$,

$$
H_{d}(x)=c_{d} x^{-\alpha} \frac{x^{\rho}-1}{\rho}, \quad \text { where } \quad c_{d}=\frac{\rho 2^{\alpha}}{2^{\rho}-1} \chi\left(2\left(\nu\left(\Gamma_{d}\right)\right)^{1 / \alpha} \Gamma_{d}\right)\left(=\frac{\rho 2^{\alpha}}{2^{\rho}-1} H_{d}(2)\right) .
$$

Hence $S_{d} \in 2 \mathcal{R} \mathcal{V}_{-\alpha, \rho}\left(b_{d}, A_{d}, H_{d}\right)$, as claimed. 


\subsection{Examples of different degeneracies under second order regular variation}

In this section we provide examples of a couple of degeneracies that come up while trying to connect second order regular variation in the multivariate and the univariate case. Suppose $X_{1}, X_{2}, \ldots, X_{n}$ are iid $2 \mathcal{R} \mathcal{V}_{-\alpha, \rho}$. Then using (Mao and Hu, 2013, Theorem 3.1, 3.2), we know that the sum $\sum_{i=1}^{n} X_{i} \in$ $2 \mathcal{R} \mathcal{V}_{-\alpha, \rho}$. But can we say $\boldsymbol{X} \in 2 \mathcal{M R} \mathcal{V}$ ? This may not always be true as we see in the following example.

Example 2.1. Suppose $X_{1}, X_{2}$ are iid random variables with distribution function $F$ such that

$$
\bar{F}(x)=\frac{1}{2} x^{-\alpha}\left(1+x^{\rho}\right), \quad x \geq 1,
$$

where $\alpha>0, \rho<0$. This family of distributions belongs to the Hall-Welsh class of heavy-tailed distributions. For any $\alpha>0$ and $\rho<0$, it is immediate that $X_{1} \in 2 \mathcal{R} \mathcal{V}_{-\alpha, \rho}(b, A)$ where $b(t)=t^{1 / \alpha}$ and $A(t)=t^{\rho}$. Take a set of the form $\left[0,\left(x_{1}, x_{2}\right)\right]^{c}$ for $x_{1}>0, x_{2}>0$ and observe that

$$
t \mathbb{P}\left(\frac{\boldsymbol{X}}{t^{1 / \alpha}} \in\left[0,\left(x_{1}, x_{2}\right)\right]^{c}\right) \underset{t \rightarrow \infty}{\rightarrow} \frac{1}{2}\left(\frac{1}{x_{1}^{\alpha}}+\frac{1}{x_{2}^{\alpha}}\right)=: \nu\left(\left[0,\left(x_{1}, x_{2}\right)\right]^{c}\right) .
$$

At the second level

$$
\begin{aligned}
& \frac{t \mathbb{P}\left(\frac{\boldsymbol{X}}{t^{1 / \alpha}} \in\left[0,\left(x_{1}, x_{2}\right)\right]^{c}\right)-\frac{1}{2}\left(\frac{1}{x_{1}^{\alpha}}+\frac{1}{x_{2}^{\alpha}}\right)}{t^{\rho / \alpha}} \\
= & \frac{t \mathbb{P}\left(X_{1}>t^{1 / \alpha} x_{1}\right)+t \mathbb{P}\left(X_{2}>t^{1 / \alpha} x_{2}\right)-t \mathbb{P}\left(X_{1}>t^{1 / \alpha} x_{1}, X_{2}>t^{1 / \alpha} x_{2}\right)-x_{1}^{-\alpha} / 2-x_{2}^{-\alpha} / 2}{t^{\rho / \alpha}} \\
= & \frac{1}{2}\left(x_{1}^{-\alpha+\rho}+x_{2}^{-\alpha+\rho}\right)-\frac{t^{-1-\rho / a}}{4} x_{1}^{-\alpha} x_{2}^{-\alpha}\left(1+t^{\rho / \alpha} x_{1}^{\rho}\right)\left(1+t^{\rho / \alpha} x_{2}^{\rho}\right)=H^{*}\left(x_{1}, x_{2}, t\right) . \quad \text { (say) }
\end{aligned}
$$

Now, we have

$$
\lim _{t \rightarrow \infty} H^{*}\left(x_{1}, x_{2}, t\right)= \begin{cases}\frac{1}{2}\left(x_{1}^{-\alpha+\rho}+x_{2}^{-\alpha+\rho}\right) & \text { if } \rho+\alpha>0 \\ \frac{1}{2}\left(x_{1}^{-2 \alpha}+x_{2}^{-2 \alpha}\right)-\frac{1}{4} x_{1}^{-\alpha} x_{2}^{-\alpha} & \text { if } \rho+\alpha=0 \\ -\infty & \text { if } \rho+\alpha<0\end{cases}
$$

We can check that no other choice of $A(\cdot)$ (up to equivalent tail behavior) would provide a finite limit for (2.4) as $t \rightarrow \infty$. Hence we have $\boldsymbol{X} \in 2 \mathcal{M R} \mathcal{V}_{-\alpha, \rho}$ iff $\alpha+\rho \geq 0$. Thus for any choice of $\rho$ such that $\alpha+\rho<0, X_{1} \in 2 \mathcal{R} \mathcal{V}_{-\alpha, \rho}$, but $\boldsymbol{X}$ is not $2 \mathcal{M R} \mathcal{V}$.

In the next example, we have independent (but not identically distributed) random variables $X_{1}, X_{2}$, where the marginal distributions are both $2 \mathcal{R} \mathcal{V}_{-\alpha, \rho}$, and the joint distribution is also $2 \mathcal{M} \mathcal{R} \mathcal{V}$, yet, we cannot use Proposition 2.1.

Example 2.2. Let $\boldsymbol{X}=\left(X_{1}, X_{2}\right)=B\left(Z_{1}, 0\right)+(1-B)\left(0, Z_{2}\right)$, where $B \sim \operatorname{Bernoulli}(1 / 2)$ and independent of $Z_{1}, Z_{2}$, which are independent random variables with distribution functions $F_{1}, F_{2}$ respectively, such that, for $x \geq 1$,

$$
\overline{F_{1}}(x)=\frac{1}{2} x^{-2}\left(1+x^{-1}\right) \quad \text { and } \quad \overline{F_{2}}(x)=x^{-2}\left(1-\frac{1}{2} x^{-1}+\frac{1}{2} x^{-2}\right) .
$$


Note that we created a random vector whose realizations are all on the two axes (there is no interior point). We can check that both $X_{1}, X_{2} \in 2 \mathcal{R} \mathcal{V}_{-2,-1}(b, A)$ where $b(t)=t^{1 / 2}$ and $A(t)=t^{-1}$. Take a set of the form $\left[0,\left(x_{1}, x_{2}\right)\right]^{c}$ for $x_{1}>0, x_{2}>0$ and observe that, as $t \rightarrow \infty$,

$$
t \mathbb{P}\left(\frac{\boldsymbol{X}}{t^{1 / 2}} \in\left[0,\left(x_{1}, x_{2}\right)\right]^{c}\right) \underset{t \rightarrow \infty}{\rightarrow} \frac{1}{4 x_{1}^{2}}+\frac{1}{2 x_{2}^{2}}=: \nu\left(\left[0,\left(x_{1}, x_{2}\right)\right]^{c}\right)
$$

At the second level,

$$
\frac{t \mathbb{P}\left(\frac{\boldsymbol{X}}{t^{1 / 2}} \in\left[0,\left(x_{1}, x_{2}\right)\right]^{c}\right)-\left(\frac{1}{4 x_{1}^{2}}+\frac{1}{2 x_{2}^{2}}\right)}{t^{-1 / 2}} \underset{t \rightarrow \infty}{\rightarrow} \frac{1}{4}\left(x_{1}^{-3}-x_{2}^{-3}\right)=: H\left(x_{1}, x_{2}\right)=\chi\left(\left[(0,0),\left(x_{1}, x_{2}\right)\right]^{c}\right) .
$$

Since the random vectors lie only on the axes, we have $\mathbb{P}\left(X_{1}+X_{2}>x\right)=\mathbb{P}\left(\boldsymbol{X} \in[0,(x, x)]^{c}\right)$, and we can check that for any $x>0$,

$$
\frac{t \mathbb{P}\left(\frac{\boldsymbol{X}}{t^{1 / 2}} \in x \Gamma_{2}\right)-\frac{3}{4 x^{2}}}{t^{-1 / 2}}=\frac{t \mathbb{P}\left(\frac{X_{1}+X_{2}}{t^{1 / 2}}>x\right)-\frac{3}{4 x^{2}}}{t^{-1 / 2}}=\frac{t \mathbb{P}\left(\frac{\boldsymbol{X}}{t^{1 / 2}} \in[0,(x, x)]^{c}\right)-\frac{3}{4 x^{2}}}{t^{-1 / 2}} \underset{t \rightarrow \infty}{\rightarrow} \frac{x^{-3}-x^{-3}}{4}=0=\chi\left(x \Gamma_{2}\right)
$$

Hence we can conclude that $c_{2}=0$ (as defined in (2.1)). Thus Proposition 2.1 cannot be used.

\section{Diversification index}

\subsection{Risk measures and diversification}

In risk management, evaluating diversification benefits properly is key for both insurance and investments. Indices have been introduced to quantify and compare the diversification of portfolios, such as the closely related notions of diversification benefit defined by Bürgi et al. (2008) as

$$
1-\frac{\tilde{\rho}\left(\sum_{i=1}^{d} X_{i}\right)}{\sum_{i=1}^{d} \tilde{\rho}\left(X_{i}\right)}, \quad \text { with } \tilde{\rho}(\cdot):=\rho(\cdot)-\mathbb{E}(\cdot),
$$

and the associated diversification index defined by Tasche (2008) as,

$$
D_{\rho}(\boldsymbol{X})=\frac{\rho\left(\sum_{i=1}^{d} X_{i}\right)}{\sum_{i=1}^{d} \rho\left(X_{i}\right)}
$$

for $d$ risks $\left(X_{i}, i=1, \cdots, d\right), \rho$ denoting the associated risk measure. This index $D_{\rho}(\boldsymbol{X})$ is also referred to as a measure of risk concentration by some authors. Neither index is a so-called universal risk measure and they depend on the choice of the associated risk measure $\rho$ and on the number $d$ of the underlying risks in the portfolio (see e.g. Emmer et al. (2015)). As indicated earlier, in this paper we restrict to the popular risk measure value-at-risk (VaR) as the choice for $\rho$ and obtain asymptotic results for the diversification index. For notational convenience, we define the associated quantity $Q_{1-\beta}(X)$ for $0<\beta<1$ for a random variable $X$ with disribution $F$ as

$$
Q_{1-\beta}(X)=\operatorname{VaR}_{\beta}(X):=\bar{F}^{\leftarrow}(1-\beta)=\inf \{x \in \mathbb{R}: \mathbb{P}(X>x) \leq 1-\beta\} .
$$

The diversification index associated with VaR under different assumptions on the marginal distributions and dependence structure, as well as its asymptotic limits can be found in the literature 
(see e.g. Bürgi et al. (2008), Dacorogna et al. (2015), Degen et al. (2010), Embrechts et al. (1997). We denote the diversification index $D_{\mathrm{VaR}_{\beta}}$ as $D_{\beta}$ to emphasize the role of $\beta$ in the calculation of the index. The following result was obtained under the assumption of independence and identical distribution of the marginal $X_{i}$ 's.

Lemma 3.1 (cf. Example 3.1, Embrechts et al. (2009)). Assume $X_{1}, \ldots, X_{n}$ are iid with distribution function $F$ where $\bar{F} \in \mathcal{R} \mathcal{V}_{-\alpha}$ with $\alpha>0$. Let $S_{d}:=\sum_{i=1}^{d} X_{i}$. Then

$$
\lim _{\beta \uparrow 1} D_{\beta}(\boldsymbol{X})=\lim _{\beta \uparrow 1} \frac{\operatorname{VaR}_{\beta}\left(S_{d}\right)}{d \operatorname{VaR}_{\beta}\left(X_{1}\right)}=\lim _{\gamma \downarrow 0} \frac{Q_{\gamma}\left(S_{d}\right)}{d Q_{\gamma}\left(X_{1}\right)}=d^{1 / \alpha-1} .
$$

The rate of convergence for the limit in (3.2) can be obtained by using an additional assumption of second order regular variation; see Albrecher et al. (2010), Degen et al. (2010), Mao and Hu (2013), Omey and Willekens (1986). Some studies relax the condition of independence of marginals and obtain limits as in (3.2) as well as rates of convergence; for instance Hua and Joe (2011) work under a scale-mixture dependence with second order regularly varying marginal distributions, Kortschak (2012) works under an assumption of asymptotic independence, and Tong et al. (2012) assume an Archimedean copula as the dependence structure. In this paper, we consider an alternative approach assuming that the random vector is multivariate regularly varying $(\mathcal{M R} \mathcal{V})$ as well as it possesses second order regular variation $(2 \mathcal{M R} \mathcal{V})$ in order to obtain the rate of convergence. To the best of our knowledge, this approach has not been looked at and forms a broad class containing examples with regularly varying margins (both possessing $2 \mathcal{R} \mathcal{V}$ and not possessing $2 \mathcal{R} \mathcal{V}$ ) as well as different families of dependence structures.

\subsection{Main Result}

The following result provides the rate of convergence for the diversification index $D_{\rho}(\boldsymbol{X})$ when taking VaR as a risk measure for a random vector $\boldsymbol{X}=\left(X_{1}, \ldots, X_{d}\right)$ that exhibits second order regular variation. Note that even if $\boldsymbol{X} \in 2 \mathcal{M} \mathcal{R} \mathcal{V}$, the marginal random variables $X_{i}$ need not to be $2 \mathcal{R} \mathcal{V}$. We assume that the marginals are identically distributed although not necessarily independent.

Theorem 3.2. Let $\boldsymbol{X} \in 2 \mathcal{M R} \mathcal{V}_{-\alpha, \rho}(b, A, \nu, H)$ with functions $b(t) \uparrow \infty$ and $A(t) \rightarrow 0$, as $t \rightarrow \infty$. Assume either Assumption 1 or Assumption 2 holds. From Proposition 2.1, we have $S_{d}=\sum_{i=1}^{d} X_{i} \in$ $2 \mathcal{R} \mathcal{V}_{-\alpha, \rho}\left(b_{d}, A_{d}, H_{d}\right)$ with $b_{d}, A_{d}$ and $H_{d}$ as defined in (2.1). Then, for $d \geq 2$,

$$
\lim _{\beta \uparrow 1} D_{\beta}(\boldsymbol{X})=\lim _{\beta \uparrow 1} \frac{\operatorname{VaR}_{\beta}\left(S_{d}\right)}{\sum_{i=1}^{d} \operatorname{VaR}_{\beta}\left(X_{i}\right)}=\lim _{\gamma \downarrow 0} \frac{Q_{\gamma}\left(S_{d}\right)}{d Q_{\gamma}\left(X_{1}\right)}=K_{d} \quad \text { where } \quad K_{d}:=\frac{1}{d}\left(\frac{\nu\left(\Gamma_{d}\right)}{\nu\left(\Gamma_{1}\right)}\right)^{1 / \alpha}
$$

with $\Gamma_{d}$ defined in (2.2), for any $d \geq 1$. Moreover, if

$$
\left|\chi\left(2\left(\nu\left(\Gamma_{d}\right)\right)^{1 / \alpha} \Gamma_{d}\right)\right|<\infty, \forall d \geq 1, \quad \text { and } \quad\left|\chi\left(2\left(\nu\left(\Gamma_{d}\right)\right)^{1 / \alpha} \Gamma_{d}\right)\right| \neq\left|\chi\left(2\left(\nu\left(\Gamma_{1}\right)\right)^{1 / \alpha} \Gamma_{1}\right)\right|, \forall d \geq 2,
$$

then we have, for any $x>0$,

$$
\lim _{\gamma \downarrow 0} \frac{D_{1-\gamma x}(\boldsymbol{X})-K_{d}}{A(b(1 / \gamma)))}=\lim _{\gamma \downarrow 0} \frac{\frac{Q_{\gamma x}\left(S_{d}\right)}{d Q_{\gamma x}\left(X_{1}\right)}-K_{d}}{A(b(1 / \gamma)))}=C \frac{K_{d}}{\alpha \rho}\left(x^{-\rho / \alpha}-1\right),
$$


for a constant $C \neq 0$ that is identified, for $d \geq 2$, as

$$
C= \begin{cases}c_{d}-c_{1} & \text { if } X_{1} \sim 2 R V \\ c_{d}(\neq 0) & \text { otherwise }\end{cases}
$$

with $c_{d}$ and $c_{1}$ defined in (2.1).

\section{Remark 3.3.}

(i) Note that if $\left|\chi\left(2\left(\nu\left(\Gamma_{d}\right)\right)^{1 / \alpha} \Gamma_{d}\right)\right|=\infty$, the question about the limiting rate of convergence above remains open.

(ii) Even if $c_{d} \neq 0$ and $c_{1} \neq 0$, it is possible that $C=c_{d}-c_{1}=0$; we have not found any example of this type.

In order to prove Theorem 3.2, we need the following result that is a direct application of a lemma from Vervaat (see Vervaat (1971)).

Lemma 3.4. For any positive random variable $X \in 2 \mathcal{R} \mathcal{V}_{-\alpha, \rho}(b, A)$, we have

$$
\lim _{\gamma \downarrow 0} \frac{\frac{1}{b(1 / \gamma)} Q_{\gamma x}(X)-x^{-1 / \alpha}}{A(b(1 / \gamma))}=\frac{c_{1}}{\alpha \rho} x^{-1 / \alpha}\left(x^{-\rho / \alpha}-1\right)=: H_{1}^{*}(x)
$$

with $0<c_{1}<\infty$ defined in (2.1).

Example 3.1. The following example is an application of Lemma 3.4. Suppose $X \sim F$, where $\bar{F}(x)=1-F(x)=\frac{1}{2}\left(x^{-\alpha}+x^{-2 \alpha}\right)$. Hence, for $0<p<1, \bar{F}^{\leftarrow}(p)=2^{1 / \alpha}(\sqrt{1+8 p}-1)^{-1 / \alpha}$. With $b(t)=\bar{F}^{\leftarrow}(1 / t)$ we have, for $x>0$, $t \bar{F}(b(t) x)=\frac{t}{2}\left[\frac{1}{2}\left(\sqrt{1+\frac{8}{t}}-1\right) x^{-\alpha}+\frac{1}{4}\left(\sqrt{1+\frac{8}{t}}-1\right)^{2} x^{-2 \alpha}\right]=x^{-\alpha}\left(1+\frac{2}{t}\left(x^{-\alpha}-1\right)+o\left(t^{-1}\right)\right) \underset{t \rightarrow \infty}{\rightarrow} x^{-\alpha}$. Moreover, taking $A(t)=t^{-\alpha}$, we have $A(b(t))=\frac{2}{t}\left[1-\frac{2}{t}+o(1 / t)\right]$, from which we deduce that

$$
\lim _{t \rightarrow \infty} \frac{t \bar{F}(b(t) x)-x^{-\alpha}}{A(b(t))}=x^{-\alpha}\left(x^{-\alpha}-1\right)=: H(x) .
$$

Hence, $X \in 2 \mathcal{R} \mathcal{V}_{-\alpha,-\alpha}(b, A, H)$ with $c=-\alpha$ as defined in (1.2). Applying Lemma 3.4, we have

$$
\lim _{\gamma \downarrow 0} \frac{\frac{1}{b(1 / \gamma)} Q_{\gamma x}(X)-x^{-1 / \alpha}}{A(b(1 / \gamma))}=\frac{1}{\alpha} x^{-1 / \alpha}(x-1),
$$

which can also be directly verified. Note that $Q_{\gamma x}(X)=\operatorname{VaR}_{1-\gamma x}(X)=b(1 /(\gamma x))$.

Proof of Lemma 3.4. The proof is an application of Vervaat's Lemma that we recall here for the sake of completeness. 
Vervaat's Lemma (see Vervaat (1971)). Suppose y is a continuous function on $[0, \infty)$ and $\left\{z_{t}(x)\right\}_{t \geq 0}$ is a family of non-negative, non-increasing functions. Also assume that the function $g$ has a negative continuous derivative. Let $\delta(t) \rightarrow 0$ with $\delta(t)>0$ eventually and

$$
\lim _{t \rightarrow \infty} \frac{z_{t}(x)-g(x)}{\delta(t)}=y(x)
$$

locally uniformly on $(0, \infty)$. Then, locally uniformly on $(g(0), g(\infty))$,

$$
\lim _{t \rightarrow \infty} \frac{z_{t}^{\leftarrow}(x)-g^{\leftarrow}(x)}{\delta(t)}=-\left(g^{\leftarrow}\right)^{\prime}(x) y\left(g^{\leftarrow}(x)\right)
$$

Let $\gamma=1 / t$, so that $\gamma \downarrow 0$ as $t \rightarrow \infty$. Applying Vervaat's Lemma with $z_{t}(x)=t \mathbb{P}[X>x b(t)]=$ $t \bar{F}_{X}(x b(t)), g(x)=x^{-\alpha}, \delta(t)=A(b(t))$ and $y(x)=H_{1}(x)$ given in (2.1), we obtain:

$$
\lim _{t \rightarrow \infty} \frac{z_{t}^{\leftarrow}(x)-g^{\leftarrow}(x)}{\delta(t)}=\lim _{t \rightarrow \infty} \frac{\frac{1}{b(t)} \bar{F}_{X}^{\leftarrow}(x / t)-x^{-1 / \alpha}}{A(b(t))}=\lim _{\gamma \downarrow 0} \frac{\frac{1}{b(1 / \gamma)} Q_{\gamma x}(X)-x^{-1 / \alpha}}{A(b(1 / \gamma))},
$$

hence the result given in Lemma 3.4.

Proof of Theorem 3.2. Since $\boldsymbol{X} \in 2 \mathcal{M R \mathcal { V }}_{-\alpha, \rho}(b, A, \nu, H)$, and $X_{i}$ 's are identically distributed, if $X_{i} \sim F$ then $\bar{F} \in \mathcal{R} \mathcal{V}_{-\alpha}$. Proposition 2.1 (with the same notations) provides that $S_{d}$ is $2 R V_{-\alpha, \rho}\left(b_{d}, A_{d}, H_{d}\right)$ such that, for $x>0, \frac{t \mathbb{P}\left[S_{d}>b_{d}(t) x\right]-x^{-\alpha}}{A_{d}\left(b_{d}(t)\right)} \underset{t \rightarrow \infty}{\longrightarrow} H_{d}(x)$. Applying Lemma 3.4 for $S_{d}$ gives then

$$
\lim _{\gamma \downarrow 0} \frac{\frac{1}{b_{d}(1 / \gamma)} Q_{\gamma x}\left(S_{d}\right)-x^{-1 / \alpha}}{A_{d}\left(b_{d}(1 / \gamma)\right)}=\frac{c_{d}}{\alpha \rho} x^{-1 / \alpha}\left(x^{-\rho / \alpha}-1\right)=: H_{d}^{*}(x), \quad \text { with } 0<c_{d}<\infty .
$$

First of all, since $\bar{F} \in \mathcal{R} \mathcal{V}_{-\alpha}$, introducing the notation $b_{1}$ when looking at any $X_{i}$, we can write $\lim _{\beta \uparrow 1} D_{\beta}(\boldsymbol{X})=\lim _{\beta \uparrow 1} \frac{\operatorname{VaR}_{\beta}\left(S_{d}\right)}{\sum_{i=1}^{d} \operatorname{VaR}_{\beta}\left(X_{i}\right)}=\lim _{\gamma \downarrow 0} \frac{Q_{\gamma}\left(S_{d}\right)}{d Q_{\gamma}(X)}=\lim _{\gamma \downarrow 0} \frac{1}{d} \times \frac{Q_{\gamma}\left(S_{d}\right)}{b_{d}(1 / \gamma)} \times \frac{b_{1}(1 / \gamma)}{Q_{\gamma}\left(X_{1}\right)} \times \frac{b_{d}(1 / \gamma)}{b_{1}(1 / \gamma)}=\frac{1}{d}\left(\frac{\nu\left(\Gamma_{d}\right)}{\nu\left(\Gamma_{1}\right)}\right)^{1 / \alpha}=K_{d}$.

Now, to assess the second order property, observe that for any $x>0$,

$$
\frac{D_{1-\gamma x}(\boldsymbol{X})-K_{d}}{A(b(1 / \gamma)))}=\frac{\frac{Q_{\gamma x}\left(S_{d}\right)}{d Q_{\gamma x}(X)}-K_{d}}{A(b(1 / \gamma)))}=I(x, \gamma)-I I(x, \gamma)
$$

where

$$
I(x, \gamma)=K_{d} \frac{b_{1}(1 / \gamma)}{Q_{\gamma x}\left(X_{1}\right)} \frac{\left[\frac{Q_{\gamma x}\left(S_{d}\right)}{b_{d}(1 / \gamma)}-x^{-1 / \alpha}\right]}{A(b(1 / \gamma))}
$$

and

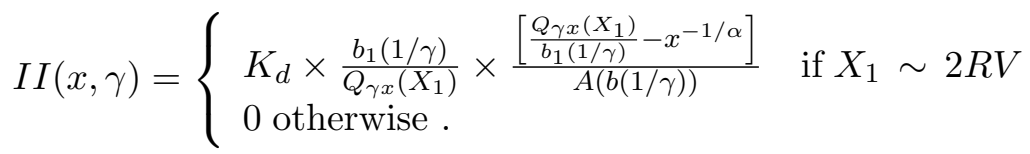


Indeed, if $X_{1}$ is $2 \mathrm{RV}$, then we have $\frac{t \mathbb{P}\left(X_{1}>b_{1}(t) x\right)-x^{-\alpha}}{A_{1}\left(b_{1}(t)\right)} \underset{t \rightarrow \infty}{\longrightarrow} H_{1}(x):=c_{1} x^{-\alpha} \frac{x^{\rho}-1}{\rho}$ with $c_{1}=$ $\frac{\rho 2^{\alpha} H_{1}(2)}{2^{\rho}-1}$ and $H_{1}(2)=\chi\left(2\left(\nu\left(\Gamma_{1}\right)\right)^{1 / \alpha} \Gamma_{1}\right)$. Moreover $b_{1}(t)=\left(\nu\left(\Gamma_{1}\right)\right)^{1 / \alpha} b(t), A_{1}(t)=A\left(\left(\nu\left(\Gamma_{1}\right)\right)^{-1 / \alpha} t\right)$, and, by construction,

$$
A(b(t))=A_{d}\left(b_{d}(t)\right)=A_{1}\left(b_{1}(t)\right) .
$$

Note that we also used the fact that $d K_{d}=b_{d}(t) / b_{1}(t)$ (for any $t>0$ ), when writing the expression of the ratio $\frac{D_{1-\gamma x}(\boldsymbol{X})-K_{d}}{A(b(1 / \gamma))}=\frac{\frac{Q_{\gamma x}\left(S_{d}\right)}{d Q_{\gamma x}(X)}-K_{d}}{A(b(1 / \gamma)))}$.

Now, we obtain via $(3.5)$, that

$$
\lim _{\gamma \downarrow 1} I(x, \gamma)=K_{d} \cdot x^{1 / \alpha} \cdot H_{d}^{*}(x)=K_{d} \frac{c_{d}}{\alpha \rho}\left(x^{-\rho / \alpha}-1\right)
$$

Similarly, when $X_{1}$ is $2 \mathrm{RV}$, applying Lemma 3.4 for $X_{1}$ gives

$$
\lim _{\gamma \downarrow 0} \frac{\frac{1}{b_{1}(1 / \gamma)} Q_{\gamma x}\left(X_{1}\right)-x^{-1 / \alpha}}{A_{1}\left(b_{1}(1 / \gamma)\right)}=\frac{c_{1}}{\alpha \rho} x^{-1 / \alpha}\left(x^{-\rho / \alpha}-1\right)=: H_{1}^{*}(x), \quad \text { with } 0<c_{1}<\infty .
$$

from which we deduce that

$$
\lim _{\gamma \downarrow 1} I I(x, \gamma)=K_{d} \cdot x^{1 / \alpha} \cdot H_{1}^{*}(x)=K_{d} \frac{c_{1}}{\alpha \rho}\left(x^{-\rho / \alpha}-1\right) .
$$

Hence (3.4) holds and the theorem is proved.

In the subsequent section we provide examples for both cases where $C=c_{d}$ and when $C=c_{d}-c_{1}$. Note that proportional growth rate of $D_{1-\gamma}(X)$ can be deduced immediately from Theeorem 3.2 providing the following corollary.

Corollary 3.5. Under the conditions of Theorem 3.2, we have, for any $x>0, y>0$,

$$
\lim _{\gamma \downarrow 0} \frac{D_{1-\gamma x}(\boldsymbol{X})-D_{1-\gamma}(\boldsymbol{X})}{D_{1-\gamma y}(\boldsymbol{X})-D_{1-\gamma}(\boldsymbol{X})}=\frac{x^{-\rho / \alpha}-1}{y^{-\rho / \alpha}-1}
$$

Under the assumption that we can statistically estimate $D_{\beta}$ at moderately high values of $\beta$, Corollary 3.5 may provide a way to extrapolate values of $D_{\beta}$ to extreme levels of $\beta$. For instance, let $\boldsymbol{X} \in 2 \mathcal{M R \mathcal { V }} \mathcal{L}_{-\alpha, \rho}$ and suppose our data allows us to compute estimates of the diversification index for VaR at $90 \%$ and $95 \%$ which is given by $\hat{D}_{0.90}(\boldsymbol{X})$ and $\hat{D}_{0.95}(\boldsymbol{X})$, then for any $p \gg 0.95$ (with $0<p<1$ ), we can use Corollary 3.5 to estimate $D_{p}(\boldsymbol{X})$ as

$$
\hat{D}_{p}(\boldsymbol{X})=\hat{D}_{0.90}(\boldsymbol{X})+\left(\frac{\left(\frac{1-p}{0.1}\right)^{-\rho / \alpha}-1}{(0.5)^{-\rho / \alpha}-1}\right)\left[\hat{D}_{0.95}(\boldsymbol{X})-\hat{D}_{0.90}(\boldsymbol{X})\right]
$$




\section{Examples}

In this section we apply Theorem 3.2 in different examples possessing $2 \mathcal{M R \mathcal { V }}$ to compute the asymptotic limit for the diversification index $D_{\beta}$; our examples are carried out in dimension $d=2$ for convenience.

In both Examples 4.1 and 4.2, the dependence structure is given by a survival Clayton copula that exhibits asymptotic dependence, hence the conditions of Assumption 1 hold. Example 4.1 additionally possesses $2 \mathcal{R} \mathcal{V}$ across the marginal distributions, whereas Example 4.2 does not. In Example 4.3, we discuss a general class of distributions possessing hidden regular variation that exhibits asymptotic independence and Assumption 2 is satisfied.

Example 4.1 (Pareto-Lomax marginal distribution with survival Clayton copula).

Suppose $\boldsymbol{X}=\left(X_{1}, X_{2}\right) \sim F$ with identical $(\alpha, 1)$-Pareto-Lomax marginal distributions, with $\alpha>1$, s.t.

$$
\bar{F}_{1}(x)=\bar{F}_{2}(x)=(1+x)^{-\alpha}, \quad \forall x>0,
$$

and that the dependence structure of $\boldsymbol{X}$ is given by a survival Clayton copula on $[0,1]^{2}$, with parameter $\theta>0$ :

$$
\mathbb{P}\left[X_{1}>x_{1}, X_{2}>x_{2}\right]=\left[\left(\bar{F}_{1}\left(x_{1}\right)\right)^{\theta}+\left(\bar{F}_{2}\left(x_{2}\right)\right)^{\theta}-1\right]^{-1 / \theta}=\left[\left(1+x_{1}\right)^{\alpha \theta}+\left(1+x_{2}\right)^{\alpha \theta}-1\right]^{-1 / \theta} .
$$

STEP 1: It has been already shown in Example 1.1 that $X_{1} \in 2 \mathcal{R} \mathcal{V}_{-\alpha,-1}\left(b, A_{1}, H\right)$ with $b(t)=t^{1 / \alpha}-1, A_{1}(t)=(t+1)^{-1}, H(x)=-\alpha x^{-\alpha}\left(x^{-1}-1\right)$, and $c=c_{1}=\alpha$. Applying Lemma 3.4 provides

$$
\lim _{\gamma \downarrow 0} \frac{\frac{\operatorname{VaR}_{1-\gamma x}\left(X_{1}\right)}{(1 / \gamma)^{1 / \alpha}-1}-x^{-1 / \alpha}}{\gamma^{1 / \alpha}}=\lim _{\gamma \downarrow 0} \frac{\frac{Q_{\gamma x}\left(X_{1}\right)}{(1 / \gamma)^{1 / \alpha}-1}-x^{-1 / \alpha}}{\gamma^{1 / \alpha}}=x^{-1 / \alpha}-1 .
$$

SteP 2: Now we verify that $\boldsymbol{X}$ is $2 \mathcal{M R \mathcal { V }}$ and identify the right parameters. We have

$$
t \mathbb{P}\left(\frac{\boldsymbol{X}}{b(t)} \in\left(\left[0, x_{1}\right] \times\left[0, x_{2}\right]\right)^{c}\right) \underset{t \rightarrow \infty}{\rightarrow} x_{1}^{-\alpha}+x_{2}^{-\alpha}-\left(x_{1}^{\alpha \theta}+x_{2}^{\alpha \theta}\right)^{-1 / \theta}=: \nu\left(\left(\left[0, x_{1}\right] \times\left[0, x_{2}\right]\right)^{c}\right) .
$$

Choosing $A(t)=-(t+1)^{-\min (\alpha \theta, 1)}$, we have

$$
\begin{aligned}
& \lim _{t \rightarrow \infty} \frac{t \mathbb{P}\left(\frac{\boldsymbol{X}}{b(t)} \in\left(\left(\left[0, x_{1}\right] \times\left[0, x_{2}\right]\right)^{c}\right)-\nu\left(\left(\left[0, x_{1}\right] \times\left[0, x_{2}\right]\right)^{c}\right)\right.}{A(b(t))}=H\left(x_{1}, x_{2}\right), \text { with } \\
& H\left(x_{1}, x_{2}\right):=\left\{\begin{array}{cc}
\frac{1}{\theta}\left(x_{1}^{\alpha \theta}+x_{2}^{\alpha \theta}\right)^{-1-\frac{1}{\theta}} & \text { if } \theta<1 / \alpha \\
\alpha\left[\left(x_{1}+x_{2}\right)^{-(\alpha+1)}\left(x_{1}+x_{2}-1\right)-x_{1}^{-(\alpha+1)}\left(x_{1}-1\right)-x_{2}^{-(\alpha+1)}\left(x_{2}-1\right)\right] & \text { if } \theta=1 / \alpha \\
\alpha\left[\left(x_{1}^{\alpha \theta}+x_{2}^{\alpha \theta}\right)^{-1-\frac{1}{\theta}}\left[x_{1}^{\alpha \theta-1}\left(x_{1}-1\right)+x_{2}^{\alpha \theta-1}\left(x_{2}-1\right)\right]\right. & \text { if } \theta>1 / \alpha \\
\left.-x_{1}^{-(\alpha+1)}\left(x_{1}-1\right)-x_{2}^{-(\alpha+1)}\left(x_{2}-1\right)\right] & \theta
\end{array}\right.
\end{aligned}
$$


from which we deduce that

$$
\boldsymbol{X} \in 2 \mathcal{M R} \mathcal{V}_{-\alpha,-1}(b, A, \nu, H) \text { with }\left\{\begin{array}{l}
b(t)=t^{1 / \alpha}-1 \\
A(t)=-(t+1)^{-\min (\alpha \theta, 1)} \\
\nu \text { defined in }(4.2) \\
H \text { defined in (4.3) }
\end{array}\right.
$$

For the next steps, we compute the density function $f$ of the distribution function $F$, as well as the density function $\lambda$ of the limit measure $\nu$, aand obtain:

$$
f\left(x_{1}, x_{2}\right)=\alpha^{2}(1+\theta)\left(1+x_{1}\right)^{\alpha \theta-1}\left(1+x_{2}\right)^{\alpha \theta-1}\left(\left(1+x_{1}\right)^{\alpha \theta}+\left(1+x_{2}\right)^{\alpha \theta}-1\right)^{-\frac{1}{\theta}-2}
$$

and

$$
\lambda\left(x_{1}, x_{2}\right)=\alpha^{2}(1+\theta) x_{1}^{\alpha \theta-1} x_{2}^{\alpha \theta-1}\left(x_{1}^{\alpha \theta}+x_{2}^{\alpha \theta}\right)^{-\frac{1}{\theta}-2} .
$$

STEP 3: We check that Assumption 1 holds. This boils down to verifying conditions (6.4)-(6.6). We do this for the case $\alpha \theta=1$, the alternative case $(\alpha \theta \neq 1)$ is analogous but is skipped for this part. Hence (4.5) and (4.6) simplify to

$$
f\left(x_{1}, x_{2}\right)=\alpha(\alpha+1)\left(1+x_{1}+x_{2}\right)^{-(\alpha+2)} \quad \text { and } \quad \lambda\left(x_{1}, x_{2}\right)=\alpha(\alpha+1)\left(x_{1}+x_{2}\right)^{-(\alpha+2)} .
$$

We have, for any $\boldsymbol{x} \in \mathbb{E}$,

$$
\frac{f(t \boldsymbol{x})}{t^{-2} \bar{F}_{1}(t)}-\lambda(\boldsymbol{x})=\lambda(\boldsymbol{x}) t^{-1}\left(\alpha-\frac{2+\alpha}{x_{1}+x_{2}}\right) \underset{t \rightarrow \infty}{\longrightarrow} 0 .
$$

Therefore, (6.4) holds and from the form of $\frac{f(t \boldsymbol{x})}{t^{-2} \bar{F}_{1}(t)}-\lambda(\boldsymbol{x})$, it is clearly bounded if $\lambda(\boldsymbol{x})$ is, which is true for $\boldsymbol{x} \in \aleph$. Thus uniform convergence also holds. Conditions (6.5) and (6.6) can also be checked in the exact same way.

SteP 4: Now, since the conditions are satisfied, applying Proposition 2.1 gives us:

$$
S_{2}=X_{1}+X_{2} \in 2 \mathcal{R} \mathcal{V}_{-\alpha,-1}\left(b_{2}, A_{2}\right)
$$

with $b_{2}(t)=\left(\nu\left(\Gamma_{2}\right)\right)^{1 / \alpha} b(t)$ and $A_{2}(t)=A\left(\left(\nu\left(\Gamma_{2}\right)\right)^{-1 / \alpha} t\right)$. Using Definition 1.2 of 2RV with (2.1), we may then conclude that

$$
\frac{t \mathbb{P}\left[S_{2} / b_{2}(t)>x\right]-x^{-\alpha}}{A_{2}\left(b_{2}(t)\right)} \underset{t \rightarrow \infty}{\rightarrow} H_{2}(x)=c_{2} x^{-\alpha}\left(1-x^{-1}\right)
$$

where $c_{2}=2^{\alpha+1} H_{2}(2)=2^{\alpha+1} \chi\left(2\left(\nu\left(\Gamma_{2}\right)\right)^{1 / \alpha} \Gamma_{2}\right)$.

STEP 5: The result on risk concentration follows by applying Theorem 3.2 (see (3.4)). For any $x>0$,

$$
\lim _{\gamma \downarrow 0} \frac{D_{1-\gamma x}(\boldsymbol{X})-K_{2}}{A_{2}\left(b_{2}(1 / \gamma)\right)}=\frac{1}{\alpha}\left(c_{1}-c_{2}\right) K_{2}\left(x^{1 / \alpha}-1\right),
$$


$A_{2}$ and $b_{2}$ being defined in Step 4. We have seen in Step 1 that $c_{1}=\alpha$. The quantities $K_{2}=$ $\frac{1}{2}\left(\frac{\nu\left(\Gamma_{2}\right)}{\nu\left(\Gamma_{1}\right)}\right)^{1 / \alpha}$ and $c_{2}$ can be computed with varying degrees of effort depending on the values of $\alpha$ and $\theta$. We show this in the next step.

SteP 6(A): $\alpha \theta=1$ First compute $\nu\left(\Gamma_{2}\right)$, using (4.7), as

$$
\nu\left(\Gamma_{2}\right)=\int_{\Gamma_{2}} \lambda\left(x_{1}, x_{2}\right) \mathrm{d} x_{1} \mathrm{~d} x_{2}=\alpha(\alpha+1) \int_{\Gamma_{2}}\left(x_{1}+x_{2}\right)^{-(\alpha+2)} \mathrm{d} x_{1} \mathrm{~d} x_{2}=\alpha+1 .
$$

Differentiating $H$ given in (4.3) w.r.t. the 2 variables, we obtain the density $h$ given by

$$
h\left(x_{1}, x_{2}\right):=\alpha^{2}(\alpha+1)\left(x_{1}+x_{2}\right)^{-(\alpha+2)}-\alpha(\alpha+1)(\alpha+2)\left(x_{1}+x_{2}\right)^{-(\alpha+3)},
$$

and can compute $\chi(\cdot)$, setting $k=2\left(\nu\left(\Gamma_{2}\right)\right)^{1 / \alpha}=2(1+\alpha)^{1 / \alpha}$, as

$$
\chi\left(k \Gamma_{2}\right)=\int_{x_{1}+x_{2}>k} h\left(x_{1}, x_{2}\right) \mathrm{d} x_{1} \mathrm{~d} x_{2}=\alpha 2^{-\alpha}\left[1-\frac{\alpha+2}{2(\alpha+1)^{1+1 / \alpha}}\right] .
$$

We deduce that $c_{2}=\alpha\left(2-(\alpha+2)(\alpha+1)^{-\left(1+\frac{1}{\alpha}\right)}\right)$. Moreover we have $K_{2}=\frac{1}{2}\left(\frac{\nu\left(\Gamma_{2}\right)}{\nu\left(\Gamma_{1}\right)}\right)^{1 / \alpha}=$ $\frac{1}{2}(1+\alpha)^{1 / \alpha}$, hence $(4.8)$ becomes, for any $x>0$,

$$
\lim _{\gamma \downarrow 0} \frac{D_{1-\gamma x}(\boldsymbol{X})-K_{2}}{A_{2}\left(b_{2}(1 / \gamma)\right)}=\frac{1}{2}\left[\frac{\alpha+2}{\alpha+1}-(\alpha+1)^{1 / \alpha}\right]\left(x^{1 / \alpha}-1\right) .
$$

Now noting that $A_{2}\left(b_{2}(1 / \gamma)\right)=-\gamma^{1 / \alpha}$, we have via $(3.4)$,

$$
\lim _{\gamma \downarrow 0} \frac{1}{\gamma^{1 / \alpha}}\left[\frac{\operatorname{VaR}_{1-\gamma x}\left(S_{2}\right)}{\operatorname{VaR}_{1-\gamma x}\left(X_{1}\right)}-(1+\alpha)^{1 / \alpha}\right]=\left[(\alpha+1)^{1 / \alpha}-\frac{\alpha+2}{\alpha+1}\right]\left(x^{1 / \alpha}-1\right) .
$$

STEP 6(B): $\alpha \theta \neq 1$ First we compute $\nu\left(\Gamma_{2}\right)$ using (4.6):

$$
\nu\left(\Gamma_{2}\right)=\int_{\Gamma_{2}} \lambda\left(x_{1}, x_{2}\right) \mathrm{d} x_{1} \mathrm{~d} x_{2}=\alpha^{2}(1+\theta) \int_{\Gamma_{2}} x_{1}^{\alpha \theta-1} x_{2}^{\alpha \theta-1}\left(x_{1}^{\alpha \theta}+x_{2}^{\alpha \theta}\right)^{-\frac{1}{\theta}-2} \mathrm{~d} x_{1} \mathrm{~d} x_{2} .
$$

This quantity can be easily numerically evaluated for specific values of $\alpha$ and $\theta$, for instance using Mathematica. Next we compute $\chi\left(k \Gamma_{2}\right)$ from which we can deduce $c_{2}=2^{\alpha+1} \chi\left(2\left(\nu\left(\Gamma_{2}\right)\right)^{1 / \alpha} \Gamma_{2}\right)$. For instance, considering the case $\alpha \theta<1$ in (4.3), and differentiating $H$ w.r.t. the 2 variables, we obtain the density $h$ given by

$$
h\left(x_{1}, x_{2}\right):=\alpha^{2}(\theta+1)(2+1 / \theta) x_{1}^{-2 \alpha \theta-1-\alpha} x_{2}^{\alpha \theta-1}\left(1+\left(x_{2} / x_{1}\right)^{\alpha \theta}\right)^{-3-1 / \theta},
$$

from which we deduce, with the change of variables $(u, v)=\left(x_{1}, x_{2} / x_{1}\right)$, and denoting $k=$ $2\left(\nu\left(\Gamma_{2}\right)\right)^{1 / \alpha}$, 


$$
\begin{aligned}
\chi\left(k \Gamma_{2}\right) & =\int_{x_{1}+x_{2}>k} h\left(x_{1}, x_{2}\right) \mathrm{d} x_{1} \mathrm{~d} x_{2} \\
& =\alpha^{2}(\theta+1)(2+1 / \theta) \int_{0}^{\infty} \int_{0}^{\infty} 1_{(u(1+v)>k)} u^{-\alpha(\theta+1)-1} v^{\alpha \theta-1}\left(1+v^{\alpha \theta}\right)^{-3-1 / \theta} \mathrm{d} v \mathrm{~d} u .
\end{aligned}
$$

This quantity can also similarly be numerically evaluated. Hence we are able to compute $c_{2}, K_{2}, A_{2}$ and $b_{2}$, and impute them into (4.8) to obtain an exact result.

Example 4.2 (Pareto-Type 1 marginal distribution with survival Clayton copula).

Here we consider the same structure of dependence as in Example 4.1, i.e. a survival Clayton copula with parameter $\theta>0$, but assume that the marginal distributions are Pareto-Type 1 marginal distributions with parameter $\alpha>1$, such that $\bar{F}_{1}(x)=\bar{F}_{2}(x)=x^{-\alpha}$ for $x>1$, to illustrate the situation where the $X_{i}$ 's are not $2 \mathrm{RV}$. For computational simplicity let $\theta=1 / \alpha$, so that, for $x_{1}>0, x_{2}>0$,

$$
\mathbb{P}\left[X_{1}>x_{1}, X_{2}>x_{2}\right]=\left(x_{1}+x_{2}-1\right)^{-\alpha} .
$$

StEP 1: First we verify that $\boldsymbol{X}$ is $2 \mathcal{M R \mathcal { V }}$ and identify the right parameters and functions. Choosing $b(t)=\left(1 / \bar{F}_{1}\right)^{\leftarrow}(t)=t^{1 / \alpha}$ and using (4.11), we observe that, for $x_{1}>0, x_{2}>0$, and for $t$ large enough such that $x_{i} t^{1 / \alpha}>1(i=1,2)$,

$$
\begin{aligned}
t \mathbb{P}\left(\frac{\boldsymbol{X}}{b(t)} \in\left(\left[0, x_{1}\right] \times\left[0, x_{2}\right]\right)^{c}\right) & =x_{1}^{-\alpha}+x_{2}^{-\alpha}-\left(x_{1}+x_{2}-t^{-1 / \alpha}\right)^{-\alpha} \\
& \rightarrow \underset{t \rightarrow \infty}{\rightarrow} x_{1}^{-\alpha}+x_{2}^{-\alpha}-\left(x_{1}+x_{2}\right)^{-\alpha}=: \nu\left(\left(\left[0, x_{1}\right] \times\left[0, x_{2}\right]\right)^{c}\right)
\end{aligned}
$$

We can also find the density function for the measure $\nu$ at $x_{1}>0, x_{2}>0$, namely

$$
\lambda\left(x_{1}, x_{2}\right)=\left|\frac{\partial^{2}}{\partial x_{1} \partial x_{2}} \nu\left(\left(\left[0, x_{1}\right] \times\left[0, x_{2}\right]\right)^{c}\right)\right|=\alpha(\alpha+1)\left(x_{1}+x_{2}\right)^{-(\alpha+2)},
$$

from which we deduce, for any $k>0$,

$$
\nu\left(k \Gamma_{1}\right)=\nu\left(([0, k] \times[0, \infty))^{c}\right)=k^{-\alpha} \quad \text { and } \quad \nu\left(k \Gamma_{2}\right)=\iint_{x_{1}+x_{2}>k} \lambda\left(x_{1}, x_{2}\right) \mathrm{d} x_{1} \mathrm{~d} x_{2}=(\alpha+1) k^{-\alpha} .
$$

To check that $\boldsymbol{X}$ is $2 \mathcal{M R} \mathcal{V}$, choosing $A(t)=-t^{-1}$, we observe that for $x_{1}>0, x_{2}>0$,

$$
\lim _{t \rightarrow \infty} \frac{t \mathbb{P}\left(\frac{\boldsymbol{X}}{b(t)} \in\left(\left[0, x_{1}\right] \times\left[0, x_{2}\right]\right)^{c}\right)-\nu\left(\left(\left[0, x_{1}\right] \times\left[0, x_{2}\right]\right)^{c}\right)}{A(b(t))}=\alpha\left(x_{1}+x_{2}\right)^{-(\alpha+1)}=: H\left(x_{1}, x_{2}\right) .
$$

Thus we have $\boldsymbol{X} \in 2 \mathcal{M R} \mathcal{V}_{-\alpha,-1}(b, A, \nu, H)$ where $b(t)=t^{1 / \alpha}, A(t)=-t^{-1}$ and $\nu, H$ are as defined in (4.12) and (4.14) respectively. We write $\chi\left(\left(\left[0, x_{1}\right] \times\left[0, x_{2}\right]\right)^{c}\right)=H\left(x_{1}, x_{2}\right)$, which can be considered as a signed measure with density given by

$$
h\left(x_{1}, x_{2}\right)=\alpha(\alpha+1)(\alpha+2)\left(x_{1}+x_{2}\right)^{-(\alpha+3)}, \quad x_{1}>0, x_{2}>0 .
$$


Then we can compute $\chi\left(k \Gamma_{2}\right)$ as

$$
\chi\left(k \Gamma_{2}\right)=\iint_{x_{1}+x_{2}>k} h\left(x_{1}, x_{2}\right) \mathrm{d} x_{1} \mathrm{~d} x_{2}=\alpha(\alpha+2) k^{-(\alpha+1)} .
$$

STEP 2: We check that Assumption 1 holds, so that we can use Theorem 3.2. This boils down to verifying conditions (6.4)-(6.6). Observe that the distribution function $F$ has a density function $f$ defined, for $x_{1}>0, x_{2}>0$, by

$$
f\left(x_{1}, x_{2}\right)=\alpha(\alpha+1)\left(x_{1}+x_{2}-1\right)^{-(\alpha+2)} .
$$

Therefore, for any $\boldsymbol{x} \in \mathbb{E}$, we obtain

$$
\begin{aligned}
\frac{f(t \boldsymbol{x})}{t^{-2} \bar{F}_{1}(t)}-\lambda(\boldsymbol{x}) & =\alpha(\alpha+1)\left(x_{1}+x_{2}\right)^{-(\alpha+2)}\left(\left[1-\frac{1}{t\left(x_{1}+x_{2}\right)}\right]^{-(\alpha+2)}-1\right) \\
& =\alpha(\alpha+1)(\alpha+2)\left(x_{1}+x_{2}\right)^{-(\alpha+3)} t^{-1}+o\left(t^{-1}\right) \underset{t \rightarrow \infty}{\rightarrow} 0 .
\end{aligned}
$$

Hence (6.4) holds and from the form of $\frac{f(t \boldsymbol{x})}{t^{-2} \bar{F}_{1}(t)}-\lambda(\boldsymbol{x})$, clearly it is bounded if $\lambda(\boldsymbol{x})$ is; which is true for $\boldsymbol{x} \in \aleph\}$. Thus uniform convergence also holds. Conditions (6.5) and (6.6) can also be checked in the exact same way.

STEP 3: Applying Proposition 2.1 provides

$$
S_{2}=X_{1}+X_{2} \in 2 \mathcal{R} \mathcal{V}_{-\alpha,-1}\left(b_{2}, A_{2}\right)
$$

where $b_{2}(t)=\left(\nu\left(\Gamma_{2}\right)\right)^{1 / \alpha} b(t)=(\alpha+1)^{1 / \alpha} t^{1 / \alpha}$ and $A_{2}(t)=A\left(\left(\nu\left(\Gamma_{2}\right)\right)^{-1 / \alpha} t\right)=-(\alpha+1)^{1 / \alpha} t^{-1}$. We also have from $(2.1)$,

$$
\lim _{t \rightarrow \infty} \frac{t \mathbb{P}\left[S_{2} / b_{2}(t)>x\right]-x^{-\alpha}}{A_{2}\left(b_{2}(t)\right)}=c_{2} x^{-\alpha}\left(1-x^{-1}\right)=: H_{2}(x),
$$

where, via (4.13) and (4.15), $c_{2}=2^{\alpha+1} \chi\left(2\left(\nu\left(\Gamma_{2}\right)\right)^{1 / \alpha} \Gamma_{2}\right)=\frac{\alpha(\alpha+2)}{(\alpha+1)^{1+1 / \alpha}}$.

STEP 4: The result on risk concentration follows by applying Theorem 3.2. For any $x>0$,

$$
\lim _{\gamma \downarrow 0} \frac{D_{1-\gamma x}(\boldsymbol{X})-K_{2}}{A_{2}\left(b_{2}(1 / \gamma)\right)}=c_{2} \frac{K_{2}}{-\alpha}\left(x^{1 / \alpha}-1\right)
$$

where $A_{2}, b_{2}, c_{2}$ are as defined in the previous Step 3 , and $K_{2}=\frac{1}{2}\left(\frac{\nu\left(\Gamma_{2}\right)}{\nu\left(\Gamma_{1}\right)}\right)^{1 / \alpha}=\frac{1}{2}(1+\alpha)^{1 / \alpha}$. Therefore we can rewrite (using the definitions of $\left.A_{2}, b_{2}, c_{2}, K_{2}\right)$, and noting that $A_{2}\left(b_{2}(1 / \gamma)\right)=$ $-\gamma^{1 / \alpha}$

$$
\lim _{\gamma \downarrow 0} \frac{1}{\gamma^{1 / \alpha}}\left[\frac{\operatorname{VaR}_{1-\gamma x}\left(S_{2}\right)}{\operatorname{VaR}_{1-\gamma x}\left(X_{1}\right)}-(1+\alpha)^{1 / \alpha}\right]=\frac{\alpha+2}{\alpha+1}\left(x^{1 / \alpha}-1\right) .
$$


Example 4.3 (Example with Hidden Regular Variation).

We consider a simple example of a mixture model possessing hidden regular variation (Das and Resnick, 2015, Section 3.1); many similar examples can be easily constructed. Let $\boldsymbol{X}=\left(X_{1}, X_{2}\right)$ be defined as

$$
\boldsymbol{X}=B_{1} \boldsymbol{Y}+\left(1-B_{1}\right)(V, V), \quad \text { with } \quad \boldsymbol{Y}=B_{2}\left(\xi_{1}, 0\right)+\left(1-B_{2}\right)\left(0, \xi_{2}\right)
$$

where $B_{1}, B_{2}, \xi_{1}, \xi_{2}, V$ are independent, $B_{1}, B_{2}$ are Bernoulli variables with $\mathbb{P}\left[B_{i}=1\right]=\mathbb{P}\left[B_{i}=\right.$ $0]=1 / 2, i=1,2 ; \xi_{1}, \xi_{2}$ are identical Pareto (Type-1) variables with parameter $\alpha>0$, whereas $V$ is a Pareto (Type-1) variable with parameter $2 \alpha$. Here $\boldsymbol{Y}$ concentrates on the axes and provides the top level regular variation, whereas $(V, V)$ is the source of hidden regular variation (and also second order regular variation) for $\boldsymbol{X}$. Note that for $z_{1}>1, z_{2}>1$,

$$
\mathbb{P}\left(\boldsymbol{X} \in\left(\left[0, x_{1}\right] \times\left[0, x_{2}\right]\right)^{c}\right)=\frac{1}{4}\left(x_{1}^{-\alpha}+x_{2}^{-\alpha}\right)+\frac{1}{2}\left(\min \left(x_{1}, x_{2}\right)\right)^{-2 \alpha} .
$$

Moreover, $X_{1}, X_{2} \sim F$ and, for $x>1, \bar{F}(x)=\frac{1}{4} x^{-\alpha}+\frac{1}{2} x^{-2 \alpha}$.

This is an example exhibiting asymptotic independence, hence we need to verify that Assumption 2 holds to apply Theorem 3.2.

STEP 1: For large $t$, with $b(t)=\bar{F}^{\leftarrow}(1 / t)=4^{1 / \alpha}(\sqrt{1+32 / t}-1)^{-1 / \alpha}=\left(\frac{t}{4}\right)^{1 / \alpha}\left(1-\frac{8}{t}+o(1 / t)\right)^{-1 / \alpha}$,

$$
\begin{aligned}
& t \mathbb{P}\left(\frac{\boldsymbol{X}}{b(t)} \in\left(\left[0, x_{1}\right] \times\left[0, x_{2}\right]\right)^{c}\right) \\
& =\left(1-\frac{8}{t}+o(1 / t)\right) x_{1}^{-\alpha}+\left(1-\frac{8}{t}+o(1 / t)\right) x_{2}^{-\alpha}+\frac{8}{t}\left(1-\frac{16}{t}+o(1 / t)\right)\left(\min \left(x_{1}, x_{2}\right)\right)^{-2 \alpha} \\
& \underset{t \rightarrow \infty}{\rightarrow} x_{1}^{-\alpha}+x_{2}^{-\alpha}=: \nu\left(\left(\left[0, x_{1}\right] \times\left[0, x_{2}\right]\right)^{c}\right) .
\end{aligned}
$$

Hence the first condition in Assumption 2 is satisfied. This also means that $\nu$ does not have a density and the measure concentrates on the two axes, hence we can write

$$
\nu\left(\Gamma_{2}\right)=\nu\left(\left(x_{1}, x_{2}\right) \in[0, \infty)^{2}: x_{1}+x_{2}>1\right)=1^{-\alpha}+1^{-\alpha}=2 .
$$

STEP 2: Marginally we observe that

$t \mathbb{P}\left(X_{1}>b(t) x\right)=\left(1-\frac{8}{t}+o(1 / t)\right) x^{-\alpha}+\frac{8}{t}\left(1-\frac{16}{t}+o(1 / t)\right) x^{-2 \alpha}=x^{-\alpha}+\frac{8}{t} x^{-\alpha}\left(x^{-\alpha}-1\right)+o(1 / t)$.

Choosing $A(t)=2 t^{-\alpha}$, we obtain

$$
\lim _{t \rightarrow \infty} \frac{t \mathbb{P}\left(X_{1} / b(t)>x\right)-x^{-\alpha}}{A(b(t))}=x^{-\alpha}\left(x^{-\alpha}-1\right)=c_{1} x^{-\alpha} \frac{x^{\rho}-1}{\rho},
$$

where $\rho=-\alpha$ and $c_{1}=-\alpha$. Both margins are identical, therefore the second condition in Assumption 2 also holds and we can infer convergence of signed measures for $2 \mathcal{M R} \mathcal{V}$. 
STEP 3: Now, for the same choice of functions $b$ and $A$, we can write, for $x_{1}>0, x_{2}>0$,

$$
\lim _{t \rightarrow \infty} \frac{t \mathbb{P}\left(\frac{\boldsymbol{X}}{b(t)} \in\left(\left[0, x_{1}\right] \times\left[0, x_{2}\right]\right)^{c}\right)-\nu\left(\left(\left[0, x_{1}\right] \times\left[0, x_{2}\right]\right)^{c}\right)}{A(b(t))}=H\left(x_{1}, x_{2}\right) .
$$

where

$$
\left.\left.H\left(x_{1}, x_{2}\right)=-\left(x_{1}^{-\alpha}+x_{2}^{-\alpha}\right)+\min \left(x_{1}^{-2 \alpha}, x_{2}^{-2 \alpha}\right)=:-\chi^{<}\left(\left[0, x_{1}\right] \times\left[0, x_{2}\right]\right)^{c}\right)+\chi^{>}\left(\left[0, x_{1}\right] \times\left[0, x_{2}\right]\right)^{c}\right) .
$$

STEP 4: Since $\nu\left(\Gamma_{2}\right)=2$ (Step 1), with $\chi=\chi^{>}-\chi^{<}$as defined in Theorem 6.3 in the Appendix, and using (4.19), we can compute for any $k>0$,

$$
\chi\left(k \Gamma_{2}\right)=-\left(k^{-\alpha}+k^{-\alpha}\right)+(k / 2)^{-2 \alpha}=-2 k^{-\alpha}\left(1-2^{2 \alpha-1} k^{-\alpha}\right) .
$$

Applying Proposition 2.1, we have $S_{2}=X_{1}+X_{2} \in 2 \mathcal{R} \mathcal{V}_{-\alpha,-\alpha}\left(b_{2}, A_{2}\right)$ where

$b_{2}(t)=\left(\nu\left(\Gamma_{2}\right)\right)^{1 / \alpha} b(t)=8^{1 / \alpha}(\sqrt{1+32 / t}-1)^{-1 / \alpha} \sim\left(\frac{t}{2}\right)^{1 / \alpha}$ and $\quad A_{2}(t)=A\left(\left(\nu\left(\Gamma_{2}\right)\right)^{-1 / \alpha} t\right)=A\left(2^{-1 / \alpha} t\right)=4 t^{-\alpha}$

We also have from $(2.1)$,

$$
\lim _{t \rightarrow \infty} \frac{t \mathbb{P}\left[S_{2} / b_{2}(t)>x\right]-x^{-\alpha}}{A_{2}\left(b_{2}(t)\right)}=-\frac{c_{2}}{\alpha} x^{-\alpha}\left(x^{-\alpha}-1\right)=: H_{2}(x),
$$

where

$$
c_{2}=\frac{-\alpha 2^{\alpha}}{2^{-\alpha}-1} \chi\left(2\left(\nu\left(\Gamma_{2}\right)\right)^{1 / \alpha} \Gamma_{2}\right)=\frac{\alpha 2^{2 \alpha}}{2^{\alpha}-1} \chi\left(2^{1+1 / \alpha} \Gamma_{2}\right)=\alpha 2^{\alpha} \frac{2^{\alpha-2}-1}{2^{\alpha}-1} .
$$

STEP 5: Finally we obtain the result on risk concentration by applying Theorem 3.2. For any $x>0$

$$
\lim _{\gamma \downarrow 0} \frac{D_{1-\gamma x}(\boldsymbol{X})-K_{2}}{A_{2}\left(b_{2}(1 / \gamma)\right)}=\left(c_{2}-c_{1}\right) \times \frac{K_{2}}{\left(-\alpha^{2}\right)}(x-1)=-2^{1 / \alpha-1} \frac{2^{2(\alpha-1)}-1}{\alpha\left(2^{\alpha}-1\right)}(x-1),
$$

where we have $A_{2}, b_{2}, c_{2}$ are as defined in Step 4 above and $K_{2}=\frac{1}{2}\left(\frac{\nu\left(\Gamma_{2}\right)}{\nu\left(\Gamma_{1}\right)}\right)^{1 / \alpha}=2^{1 / \alpha-1}$. Therefore we can rewrite, using the definitions of $A_{2}, b_{2}, c_{2}, K_{2}$ and noticing that $A_{2}\left(b_{2}(1 / \gamma)\right) \sim 8 \gamma$,

$$
\lim _{\gamma \downarrow 0} \frac{1}{\gamma}\left[\frac{\operatorname{VaR}_{1-\gamma x}\left(S_{2}\right)}{\operatorname{VaR}_{1-\gamma x}\left(X_{1}\right)}-2^{1 / \alpha}\right]=2^{1 / \alpha+3} \frac{2^{2(\alpha-1)}-1}{\alpha\left(2^{\alpha}-1\right)}(1-x) .
$$

\section{Conclusion}

Our goal in this paper was to exhibit the strength of the assumption of second order multivariate regular variation for understanding diversification benefits in a portfolio of risk factors. We have seen that $2 \mathcal{M R \mathcal { V }}$ encompasses a broad variety of dependence structures where we could compute the diversification index and observe penultimate behavior of portfolio of risk factors with respect to the risk measure VaR. Explicit computations of the constants in many examples seem tedious, 
although numerical tools can be often used here. A few questions still remain open. For instance, a characterization of multivariate second order regular variation in terms of linear combination of its marginals akin to a Cramér-Wold Theorem is yet to be discovered. We are also interested in finding the effects of the related concept of hidden regular variation on diversification.

Acknowledgement: Bikramjit Das gratefully acknowledges partial support from MOE-2013-T21-158. Partial support from RARE-318984 (an FP7 Marie Curie IRSES Fellowship) is kindly acknowledged by both authors.

\section{References}

H. Albrecher, C. Hipp, and D. Kortschak. Higher-order expansions for compound distributions and ruin probabilities with subexponential claims. Scandinavian Actuarial Journal, 2010(2):105-135, 2010.

S. Alink, M. Löwe, and M. V. Wüthrich. Diversification of aggregate dependent risks. Insurance Math. Econom., 35(1):77-95, 2004.

P. Barbe, A.-L. Fougères, and C. Genest. On the tail behavior of sums of dependent risks. Astin Bull., 36(2):361-373, 2006.

B. Basrak, R. Davis, and T. Mikosch. A characterization of multivariate regular variation. The Annals of Applied Probability, 12(3):908-920, 2002.

N. H. Bingham, C. M. Goldie, and J. L. Teugels. Regular variation, volume 27 of Encyclopedia of Mathematics and its Applications. Cambridge University Press, Cambridge, 1989.

J. Boman and F. Lindskog. Support theorems for the Radon transform and Cramér-Wold theorems. J. Theoret. Probab., 22(3):683-710, 2009.

R. Bürgi, M. Dacorogna, and R. Iles. Risk aggregation, dependence structure and diversification benefit. In Stress testing for financial institutions, pages 265-306. Risk Books, Incisive Media, London, 2008.

M. Dacorogna, L. Elbahtouri, and M. Kratz. Explicit diversification benefit for dependent risks. ESSEC Working Paper 1552, 2015. URL http://dx.doi.org/10.2139/ssrn. 2716093.

B. Das and S.I. Resnick. Models with hidden regular variation: generation and detection. Stochastic Systems, 5(2):195-238 (electronic), 2015.

L. de Haan. On regular variation and its applications to the weak convergence of sample extremes. Mathematical Centre Tracts Vol. 32, 1970.

L. de Haan and A. Ferreira. Extreme Value Theory: An Introduction. Springer-Verlag, New York, 2006.

L. de Haan and S.I. Resnick. Estimating the limit distribution of multivariate extremes. Communications in Statistics. Stochastic Models, 9(2):275-309, 1993.

M. Degen and P. Embrechts. Scaling of high-quantile estimators. Journal of Applied Probability, 48(4):968-983, 2011. ISSN 0021-9002.

M. Degen, D. Lambrigger, and J. Segers. Risk concentration and diversification: second-order properties. Insurance: Mathematics \& Economics, 46(3):541-546, 2010.

P. Embrechts, C. Klüppelberg, and T. Mikosch. Modelling Extreme Events for Insurance and Finance. Springer-Verlag, Berlin, 1997.

P. Embrechts, A. McNeil, and D. Straumann. Correlation and dependence in risk management: 
properties and pitfalls. In M. A. H. Dempster, editor, Risk Management: Value at Risk and Beyond, pages 176-223, Cambridge, 2002. Cambridge University Press.

P. Embrechts, D. Lambrigger, and M. Wüthrich. Multivariate extremes and the aggregation of dependent risks: examples and counter-examples. Extremes, 12(2):107-127, 2009.

S. Emmer, M. Kratz, and D. Tasche. What is the best risk measure in practice? a comparison of standard measures. Journal of Risk, 18(2):31-60, 2015.

L. Hua and H. Joe. Second order regular variation and conditional tail expectation of multiple risks. Insurance: Mathematics \& Economics, 49(3):537-546, 2011.

H. Hult and F. Lindskog. On Kesten's counterexample to the Cramér-Wold device for regular variation. Bernoulli, 12(1):133-142, 2006. ISSN 1350-7265.

R. Ibragimov, D. Jaffee, and J. Walden. Diversification disasters. Journal of Financial Economics, 99(2):333-348, 2011.

D. Kortschak. Second order tail asymptotics for the sum of dependent, tail-independent regularly varying risks. Extremes, 15(3):353-388, 2012.

M. Kratz. Normex, a new method for evaluating the distribution of aggregated heavy tailed risks. application to risk measures. Extremes. Special issue on Extremes and Finance, 17(4):661-691, 2014.

T. Mao and T. Hu. Second-order properties of risk concentrations without the condition of asymptotic smoothness. Extremes, 16(4):383-405, 2013.

E. Omey and E. Willekens. Second order behaviour of the tail of a subordinated probability distribution. Stochastic Processes and their Applications, 21(2):339-353, 1986.

L. Peng, R. Wang, and J. Yang. Bounds for the sum of dependent risks and worst value-at-risk with monotone marginal densities. Finance and Stochastics, 17(2):395-417, 2013.

G. Puccetti and L. Rüschendorf. Sharp bounds for sums of dependent risks. Journal of Applied Probability, 50:42-53, 2013.

S.I. Resnick. Hidden regular variation, second order regular variation and asymptotic independence. Extremes, 5(4):303-336, 2002.

S.I. Resnick. Heavy Tail Phenomena: Probabilistic and Statistical Modeling. Springer Series in Operations Research and Financial Engineering. Springer-Verlag, New York, 2007.

S.I. Resnick. Extreme Values, Regular Variation and Point Processes. Springer Series in Operations Research and Financial Engineering. Springer, New York, 2008. Reprint of the 1987 original.

Y. Sun and H. Li. Tail approximation of value-at-risk under multivariate regular variation. Int. J. Oper. Res. (Taichung), 7(4):34-44, 2010.

D. Tasche. Capital allocation to business units and sub-portfolios: the Euler principle. In A. Resti, editor, Pillar II in the New Basel Accord: The Challenge of Economic Capital, pages 423-453. Risk Books, Incisive Media, London, 2008.

B. Tong, C. Wu, and W. Xu. Risk concentration of aggregated dependent risks: the second-order properties. Insurance: Mathematics \& Economics, 50(1):139-149, 2012.

W. Vervaat. Functional limit theorems for processes with positive drift and their inverses. Zeitschrift für Wahrscheinlichkeitstheorie und Verwandte Gebiete, 23:245-253, 1971.

G.B. Weller and D. Cooley. A sum characterization of hidden regular variation with likelihood inference via expectation-maximization. Biometrika, 101(1):17-36, 2014. ISSN 0006-3444. URL http://dx.doi.org/10.1093/biomet/ast046. 


\section{Appendix}

We discuss results and assumptions from (Resnick, 2002, Section 4.2) that are used in this paper for the sake of completeness. The following results provide conditions under which the second order regular variation condition of Definition 1.5 can be represented as vague convergence of measures. Assumption 1 gives the appropriate conditions when the limit measure $\nu(\cdot)$ as obtained in Defnition 1.4 has a density with respect to the Lebesgue measure; hence $\boldsymbol{X}$ is not asymptotically independent. On the other hand, Assumption 2 gives appropriate conditions when $\nu(\cdot)$ does not have a density; it means that asymptotic independence holds for the tail distribution of $\boldsymbol{X}$.

Suppose $\boldsymbol{X}$ is a $d$-dimensional non-negative random vector with distribution function $F$ and identical one-dimensional marginals $F_{1}$.

Assumption 1. We assume the following on $F$.

1. Let $F$ have a density $F^{\prime}$ such that for $b(t) \rightarrow \infty$,

$$
\lim _{t \rightarrow \infty}\left|b(t)^{d} t F^{\prime}(b(t) \boldsymbol{x})-\lambda(\boldsymbol{x})\right|=0, \boldsymbol{x} \in \mathbb{E},
$$

where $\lambda(\cdot) \neq 0$ is bounded on $\aleph$ and moreover

$$
\lim _{t \rightarrow \infty} \sup _{\boldsymbol{a} \in \aleph}\left|b(t)^{d} t F^{\prime}(b(t) \boldsymbol{a})-\lambda(\boldsymbol{a})\right|=0, \boldsymbol{x} \in \mathbb{E} .
$$

The limit function $\lambda(\boldsymbol{x})$ necessarily satisfies $\lambda(t \boldsymbol{x})=t^{-\alpha-d} \lambda(\boldsymbol{x})$. This implies from (Resnick, 2008) that there exists $V \in \mathcal{R} \mathcal{V}_{-\alpha}$ such that

$$
\lim _{t \rightarrow \infty} \frac{1-F(b(t) \boldsymbol{x})}{V(b(t))}=\int_{[\mathbf{0}, \boldsymbol{x}]^{c}} \lambda(\boldsymbol{u}) \mathrm{d} \boldsymbol{u}=\nu\left([\mathbf{0}, \boldsymbol{x}]^{c}\right), \quad \boldsymbol{x}>\mathbf{0} .
$$

Thus conditions (6.1) and (6.2) imply multivariate regular variation. Instead of conditions (6.1) and (6.2) it is sufficient to assume $\bar{F}_{1} \in \mathcal{R} \mathcal{V}_{-\alpha}$ and

$$
\lim _{t \rightarrow \infty}\left|\frac{F^{\prime}(t \boldsymbol{x})}{t^{-d} \bar{F}_{1}(t)}-\lambda(\boldsymbol{x})\right|=0, \boldsymbol{x} \in \mathbb{E}, \quad \text { and } \lim _{t \rightarrow \infty} \sup _{\boldsymbol{a} \in \aleph}\left|\frac{F^{\prime}(t \boldsymbol{a})}{t^{-d} \bar{F}_{1}(t)}-\lambda(\boldsymbol{a})\right|=0,
$$

and we can take $V=\bar{F}_{1}$.

2. Assume that the second order condition given in (1.2) holds for $\bar{F}_{1}$ so that $\bar{F}_{1} \in \mathcal{R} \mathcal{V}_{-\alpha}$ and $A \in \mathcal{R} \mathcal{V}_{\rho}, \rho \leq 0, A \rightarrow 0$ and for $\boldsymbol{x} \in \mathbb{E}$,

$$
\lim _{t \rightarrow \infty}\left|\frac{\frac{F^{\prime}(t \boldsymbol{x})}{t^{-d} \bar{F}_{1}(t)}-\lambda(\boldsymbol{x})}{A(t)}-\chi^{\prime}(\boldsymbol{x})\right|=0
$$

where $\chi^{\prime} \neq 0$ is integrable on sets bounded away from $\mathbf{0}$. We also assume uniform convergence on $\aleph$ :

$$
\lim _{t \rightarrow \infty} \sup _{\boldsymbol{a} \in \aleph}\left|\frac{\frac{F^{\prime}(t \boldsymbol{a})}{t^{-d} \bar{F}_{1}(t)}-\lambda(\boldsymbol{a})}{A(t)}-\chi^{\prime}(\boldsymbol{a})\right|=0
$$

Also assume that $\chi^{\prime}$ is finite and bounded on $\aleph$. 
Remark 6.1. For $\boldsymbol{X} \sim F$ with identical marginals $F_{1}$, assuming conditions (6.4)-(6.6) is sufficient for (6.1)-(6.3) to hold with $V=\bar{F}_{1}$.

Using $\nu$ as defined in (6.3), we define the signed measure

$$
\mu_{t}\left([\mathbf{0}, \boldsymbol{x}]^{c}\right):=\frac{t \mathbb{P}\left[\frac{\boldsymbol{X}}{b(t)} \in[\mathbf{0}, \boldsymbol{x}]^{c}\right]-\nu\left([\mathbf{0}, \boldsymbol{x}]^{c}\right)}{A(b(t))},
$$

which has a density given by

$$
\mu_{t}^{\prime}\left([\mathbf{0}, \boldsymbol{x}]^{c}\right):=\frac{t b(t)^{d} F^{\prime}(b(t) \boldsymbol{x})-\lambda(\boldsymbol{x})}{A(b(t))}, \quad \boldsymbol{x} \in[0, \infty)^{d} .
$$

Theorem 6.2 (Proposition 5, Resnick (2002)). If $\boldsymbol{X} \in[0, \infty)^{d}$ with distribution function $F$ and identical marginals $F_{1}$ satisfies Assumption 1 then

$$
\mu_{t}^{ \pm} \stackrel{v}{\rightarrow} \chi^{ \pm}, \quad \text { on } \quad \mathbb{E}
$$

where for $t>0, \mu_{t}^{+}, \mu_{t}^{-}, \chi^{+}, \chi^{-}$are positive Radon measures with $\mu_{t}=\mu_{t}^{+}-\mu_{t}^{-}$and $\chi=\chi^{+}-\chi^{-}$.

If $\boldsymbol{X} \in F$ with $\bar{F} \in \mathcal{M R} \mathcal{V}_{-\alpha}(b)$ but possesses asymptotic independence then the limit measure $\nu(\cdot)$ as obtained in (1.4) does not have a density with respect to Lebesgue measure. Hence Assumption 1 does not hold. In this case we require a different set of assumptions which are given below.

Assumption 2. We assume the following on F.

1. Suppose (1.5) holds with $\nu\left([\mathbf{0}, \boldsymbol{x}]^{c}\right)=\kappa \sum_{i=1}^{d} x_{i}^{-\alpha}$, where $\kappa$ is some constant.

2. Moreover the one dimensional marginals are identical and satisfy the second order condition as in Definition 1.2 such that we also have

$$
\mu_{t 1}^{ \pm}:=\left(\frac{t \mathbb{P}\left[\frac{X_{1}}{b(t)} \in \cdot\right]-\nu_{\alpha}(\cdot)}{A(b(t))}\right)^{ \pm} \stackrel{v}{\rightarrow} \chi_{1}^{ \pm},
$$

on $(0, \infty]$ where $\chi_{1}(x, \infty]=c x^{-\alpha} \frac{x^{\rho}-1}{\rho}$.

Theorem 6.3 (Theorem 2, Resnick (2002)). If $\boldsymbol{X} \in[0, \infty)^{d}$ with distribution function $F$ and Assumption 2 holds, then with $\mu_{t}$ as defined in (6.7) and $H$ as in (1.5), there exist non-negative Radon measures $\mu_{t}^{>}, \mu_{t}^{<}, \chi^{>}, \chi^{<}$such that

$$
\mu_{t}^{>} \stackrel{v}{\rightarrow} \chi^{>}, \quad \mu_{t}^{<} \stackrel{v}{\rightarrow} \chi^{<}
$$

where $\mu_{t}=\mu_{t}^{>}-\mu_{t}^{<}$and $H(\boldsymbol{x})=\chi^{>}\left([\mathbf{0}, \boldsymbol{x}]^{c}\right)-\chi^{<}\left([\mathbf{0}, \boldsymbol{x}]^{c}\right)$. For our purposes we take for any set $A$ in $[0, \infty)^{d}, \chi(A):=\chi^{>}(A)-\chi^{<}(A)$. 


\begin{tabular}{|c|c|c|c|c|}
\hline $\begin{array}{l}\text { ESSEC Business School } \\
3 \text { avenue Bernard-Hirsch } \\
\text { CS } 50105 \text { Cergy } \\
95021 \text { Cergy-Pontoise Cedex } \\
\text { France } \\
\text { Tel. }+33 \text { (0) } 134433000 \\
\text { www.essec.edu }\end{array}$ & $\begin{array}{l}\text { ESSEC Executive Education } \\
\text { CNIT BP } 230 \\
92053 \text { Paris-La Défense } \\
\text { France } \\
\text { Tel. + } 33 \text { (0) } 146924900 \\
\text { www.executive-education.essec.edu }\end{array}$ & $\begin{array}{l}\text { ESSEC Asia-Pacific } \\
5 \text { Nepal Park } \\
\text { Singapore } 139408 \\
\text { Tel. +65 } 68849780 \\
\text { www.essec.edu/asia }\end{array}$ & $\begin{array}{l}\text { ESSEC Africa-Atlantic } \\
\text { Plage des Nations } \\
\text { Sidi Bouknadel } \\
\text { Rabat-Salé } \\
\text { Morocco } \\
\text { Tel. +212 } \\
\text { (0)5 } 30104019 \\
\text { www.essec.edu }\end{array}$ & $\begin{array}{l}\text { ESSEC Africa-Indian Ocean } \\
\text { Royal Road, Pierrefonds } \\
\text { Mauritius } \\
\text { Tel. +230 } 4012400 \\
\text { www.essec.edu } \\
\text { www.icsia.mu }\end{array}$ \\
\hline
\end{tabular}

\section{- MOROCCO}

\section{Contacts}

Centre de Recherche

+33 (0)134433091

research.center@essec.fr

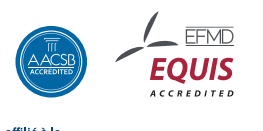

Cí CCI PARIS ILE-DE-FRANCE

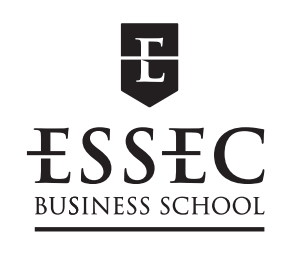

